\title{
Real-time detection of transverse stability changes in fishing vessels
}

Santiago Caamaño, Lucía; Galeazzi, Roberto; Nielsen, Ulrik D.; Míguez González, Marcos; Díaz Casás, Vicente

\section{Published in:}

Ocean Engineering

Link to article, DOI:

10.1016/j.oceaneng.2019.106369

Publication date:

2019

Document Version

Peer reviewed version

Link back to DTU Orbit

Citation (APA):

Santiago Caamaño, L., Galeazzi, R., Nielsen, U. D., Míguez González, M., \& Díaz Casás, V. (2019). Real-time detection of transverse stability changes in fishing vessels. Ocean Engineering, 189, [106369].

https://doi.org/10.1016/j.oceaneng.2019.106369

\section{General rights}

Copyright and moral rights for the publications made accessible in the public portal are retained by the authors and/or other copyright owners and it is a condition of accessing publications that users recognise and abide by the legal requirements associated with these rights.

- Users may download and print one copy of any publication from the public portal for the purpose of private study or research.

- You may not further distribute the material or use it for any profit-making activity or commercial gain

- You may freely distribute the URL identifying the publication in the public portal

If you believe that this document breaches copyright please contact us providing details, and we will remove access to the work immediately and investigate your claim 


\title{
Real-time detection of transverse stability changes in fishing vessels
}

\author{
Lucía Santiago Caamaño ${ }^{\mathrm{a}, *}$, Roberto Galeazzi ${ }^{\mathrm{b}}$, Ulrik D. Nielsen ${ }^{\mathrm{c}, \mathrm{d}}$, Marcos \\ Míguez González ${ }^{a}$, Vicente Díaz Casás ${ }^{a}$ \\ ${ }^{a}$ Integrated Group for Engineering Research, University of A Coruña, C/ Mendizbal s/n, \\ 15403 Ferrol, Spain \\ ${ }^{b}$ DTU Electrical Engineering, Technical University of Denmark, 2800 Kgs. Lyngby, \\ Denmark \\ ${ }^{c}$ DTU Mechanical Engineering, Technical University of Denmark, 2800 Kgs. Lyngby, \\ Denmark \\ ${ }^{d}$ Centre for Autonomous Marine Operations and Systems, NTNU AMOS, 7491
}

Trondheim, Norway

\begin{abstract}
Fishing is a hazardous activity due to stability related accidents, caused many times by the crew lacking information related to the level of stability of the vessel. A possible solution is offered by stability assessment systems that can help the skipper to identify potential risks and support his decision making process.

The metacentric height is a key parameter for vessel stability and its real-time monitoring may be beneficial for alerting the crew about changes in stability. The paper proposes the design of a novel stability monitoring system that automatically detects changes in metacentric height based on estimates of the roll natural frequency solely using the measured roll angle. The core of the monitoring system is a combined estimation-detection system that exploits methods in advanced signal processing and statistical change detection to properly address issues of robustness.

To analyze the monitoring performance, a nonlinear mathematical model

\footnotetext{
*Corresponding author

Email addresses: lucia.santiago. caamano@udc.es (Lucía Santiago Caamaño), rg@elektro.dtu.dk (Roberto Galeazzi), udn@mek.dtu.dk (Ulrik D. Nielsen), marcos.miguez@udc.es (Marcos Míguez González), vicente.diaz.casas@udc.es (Vicente Díaz Casás)
} 
of a stern trawler is used to generate roll motion time series in beam irregular waves of different peak period and significant wave height. Estimation and detection results obtained on the simulated data show a convincing ability of the monitoring system in discerning between safe and unsafe sailing conditions for most of the investigated cases.

Keywords:

Fishing vessels, operational guidance, roll stability, generalized likelihood ratio test, Hilbert-Huang transform, empirical model decomposition

\section{Introduction}

\subsection{Motivation}

Commercial fishing is internationally well-known by two main facts: the large variety of ship types and heterogeneity of their arrangement, size, fishing gear and typical operation; and the high fatal injury rate which has historically affected the fishing sector. Fishing is considered as one of the most dangerous occupations worldwide, including countries such as the United States, Korea, or EU coastal areas, such as Spain or the United Kingdom (Lazakis et al., 2014; Jensen et al., 2014; Petursdottir et al., 2001; European Commission, 2009).

Accidents affecting crews of fishing vessels are of very different kinds, and because the work is usually done on deck without any protection from weather and in harsh environmental conditions, falling overboard or being struck by machinery are among the most common causes of injuries. When it comes to ship-related accidents, those caused by a stability failure leading to capsizing (such as cargo shifting, large waves, hanging weights, overloading or dynamic stability issues), are the most dangerous ones (Lucas and Case, 2018). Although capsizing is the least frequent event, it is responsible for the biggest number of losses of life at sea, as it usually takes place unexpectedly while the vessel sails in rough weather (Dickey, 2008; Oliveira-Goumas and El Houdagui, 2000; Krata, 2008; Jensen et al., 2014; Bye and Lamvik, 2007). Noteworthy that most of these casualties occur in accidents involving small and medium sized fishing vessels, mainly of lengths under 12 meters (Krata, 2008; Scarponi, 2017), which represent the vast majority of the worldwide fleet (more than an $85 \%$ of the engine-powered fishing vessels have lengths under this value (Gudmundsson, 2013)). 
Different stakeholders agree that many of these accidents occur due to a chain of events that usually commence with a human error and whose effects are amplified due to the absence of a generally applied safety culture on board (Oliveira-Goumas and El Houdagui, 2000). This situation has been historically present in the fishing sector, as fishermen assume that there is an inherent risk in their activity which cannot be avoided. Accidents are usually accepted with resignation and feeling of bad luck. The situation is worsen by the huge economical pressure fishermen are subject to, as their income depend on their catches, thus forcing them to sail and work even in very unfavourable weather conditions, thereby increasing the risk level of the activity (Bye and Lamvik, 2007; Antaõ et al., 2008; Lazakis et al., 2014).

There is also another main cause for the high accident rate, which is related to the average crew training level in vessel stability matters. It has been highlighted in different publications (Wolfson Unit, 2004) that fishing vessel skippers usually present a lack of knowledge in stability evaluation, and usually assess the stability level of their vessel in a subjective way based on previous experience. Stability booklets are present in some vessels above $12 \mathrm{~m}$ in length, however their understanding is complex and are not practical in distress situations that require a quick evaluation of vessel stability (Petursdottir et al., 2001; Deakin, 2005).

\subsection{Literature survey}

In the last two decades, it has been concluded that one plausible approach to reduce stability-related accidents is the provision of simple and intuitive stability information, which helps the skipper to evaluate the sailing condition of the vessel based on objective data. This fact has been materialized in simplified on board stability guidance systems, which provide simple and clear information about the stability of the ship, including safe loading conditions and warn about situations where there may be a higher risk level (Deakin, 2005; Womack, 2003).

One of the first approaches is the stability matrix, proposed by Womack (2003). It consists of a colour coded matrix where different loading possibilities are considered, including fish in holds, presence of free fish on deck, fuel distribution, etc. Although this stability matrix represents a good tool for the smallest vessels, it may become quite complex as the number of tanks increase in larger vessels (Deakin, 2005). Similar systems, based on the use of colour coded stability posters alone or together with the analysis of residual freeboard, have also been proposed (Scarponi, 2017; Wolfson Unit, 2004). 
In the last decade, second generation operational guidance systems have been developed. These are based on simplified computer systems and software that provide an estimation of the stability level of the ship based on the loading condition details introduced in the software by the crew during operation. One such system is that one proposed by Tello et al. (2011), which also provides some basic operational guidance based on IMO MSC Circular 1228 (International Maritime Organization, 2007). Míguez González et al. (2012) proposed an operational guidance system that combines a naval architecture software with a user-friendly interface, which provides stability information following a similar colour coded approach to the aforementioned stability posters.

All of the described alternatives rely on the use of data provided by the crew, thus introducing subjectivity and large uncertainties within the stability evaluation process. In the case of stability posters, subjectivity and uncertainty appear during the selection of the loading condition from those included in the poster which better resembles the current one, while, in the case of computer systems, they appear while introducing the loading condition details which are needed to evaluate the vessel stability.

Recently, in an attempt to maximize the ease of use and the objectivity of these guidance systems, a new group of methods has been proposed. Their main objective comprises stability evaluation by automatic real-time estimation of the vessel metacentric height $(G M)$ (Wawrzynski and Krata, 2016). Terada et al. (2016) proposed a mathematical model to estimate the vessel roll natural frequency (directly related to $G M$ ) together with the roll gyradius from the measured vessel roll motion. Terada et al. (2018) further refined this approach by using the Markov chain Monte Carlo (MCMC) method as modelling tool to achieve the same objective. The authors concluded that more validation work was needed to ensure the reliability of the obtained results. Galeazzi and Perez (2011) proposed a model-based nonlinear observer for estimating the transverse stability parameters of surface vessels. The method was tested only on simulated data and requires the knowledge of the roll hydrodynamic parameters, which may be hard to obtain.

Santiago Caamaño et al. (2018a) developed a method for real-time vessel stability assessment based on the spectral analysis of roll motion using the Fast Fourier Transform (FFT) to estimate the roll natural frequency and, thus, the vessel metacentric height. This approach was further developed to reduce the uncertainty of the obtained results by implementing a sequential application of the FFT, which together with an averaging, smoothing 
and fitting process of the obtained roll spectra increases the estimation performance. Results of $G M$ estimations for two similar stern trawlers using the latter implementation, both considering roll motion from a mathematical model (Míguez González et al., 2017) and from real sea trials (Santiago Caamaño et al., 2018b), were in the range of $[-10 \%,+20 \%]$ to the target value of $G M$. Although these results were reasonable, it was concluded that further research was needed to enhance the reliability of the proposed system.

Decision support systems for operational guidance are becoming more widespread across different fields of engineering as requirements for safety and availability tightens. The maritime sector is no different and the industry has seen the proliferation of such systems for different applications. Statistical change detection has proven to be a successful methodology for the design of condition monitoring systems whenever timely decision under uncertainty and robustness are paramount. Examples of such systems are offered by Galeazzi et al. $(2013,2015)$ for the real-time monitoring of parametric roll resonance on merchant vessels; Fang et al. (2015) for the timely detection of faults in mooring systems of offshore floating platforms; Willersrud et al. (2015) for the robust detection of drillstring washouts during drilling operation for oil and gas; Hansen and Blanke (2014) for the real-time detection of airspeed sensor faults in unmanned aerial vehicles; Ghane et al. (2018) for wear detection in the downwind main shaft bearing of a spar-type floating wind turbine; Pradhan and Gupta (2017) for the detection of incoming vessels by measuring the ambient noise to enhance security of port and offshore operations.

\subsection{Contributions and novelty}

The paper presents a novel vessel stability monitoring system that integrates advanced signal processing methods with probabilistic learning to achieve robust detection of transverse stability changes in near real-time. An estimator-detector architecture is proposed to reliably extract information about the roll natural frequency from the measured roll motion and evaluate when this frequency significantly deviates over time. The Empirical Mode Decomposition (EMD) is combined with the Hilbert-Huang Transform (HHT) to estimate the roll natural frequency associated with the vessel's loading condition. The estimates are then modeled as realizations of a Weibull distributed process and a statistical change detector is designed accordingly. The detector is complemented with a situation awareness system to inform the crew about the current stability level and how close this is to 
the critical condition. The stability monitoring system has been tested on simulated data of a stern trawler sailing in beam seas. Results clearly show the ability of tracking stability changes due to variation in a vessel's loading condition and raising a timely alarm when the critical stability condition is crossed. The amplitude of the roll motion is recognized as a critical factor for the situation awareness system to correctly discriminate among the proposed stability levels.

\section{Problem formulation}

The classical procedure to estimate the stability parameters of a vessel is performing an inclining experiment. It consists of creating a controlled heel on the vessel by moving some known weights, and then obtaining the resulting metacentric height through basic initial stability principles.

Another way to obtain the metacentric height of a vessel is to carry out a roll decay test and, from the resulting roll motion time series, obtain the roll spectrum. In calm water conditions and under no external excitation, the location of the peak of the roll spectrum coincides with the roll damped natural frequency. In the case of ships, where the damping is usually quite small, this value is very close to the vessel roll natural frequency, and both values could be considered equivalent. From this value, the metacentric height can be obtained by

$$
\omega_{0}=\sqrt{\frac{g \cdot \Delta \cdot G M}{I_{x x}+A_{44}}},
$$

where $\omega_{0}$ is the roll natural frequency, $g$ is the acceleration of the gravity, $\Delta$ is the vessel mass displacement, $I_{x x}$ is the ship transverse mass moment of inertia and $A_{44}$ is the roll added mass. If the inertia and the added mass are approximated by the Weiss formula (Krüger and Kluwe, 2008), the roll natural frequency of the vessel can be obtained as

$$
\omega_{0}=\sqrt{\frac{g \cdot G M}{k_{x x}^{2}}}
$$

with $k_{x x}$ being the roll gyradius of the ship, which is usually taken as $40 \%$ of the beam of the vessel.

The inclining experiment is the method approved by the International Maritime Organization (IMO) for estimating the vessel lightship parameters (International Maritime Organization, 2008), while the above approach is 
proposed as possible means for carrying out approximate real-time estimates of the vessel stability while sailing (International Maritime Organization, 1993). However, the results of both methodologies are only reliable when the vessel is in sheltered waters, with no waves and no wind. As it is highlighted by IMO (International Maritime Organization, 1993), this fact is especially important in the case of the latter methodology. When an external excitation such as waves and wind is present, its effect is present in the roll spectrum. In these conditions the spectrum peak frequency could deviate from the vessel roll natural frequency, and the stability estimates subsequently made could be largely uncertain.

To understand the challenges inherent to the estimation of the roll natural frequency for a vessel operating in open waters the roll motion of a fishing vessel is simulated through a one degree of freedom nonlinear model subject to an external excitation.

The model is an uncoupled one degree-of-freedom mathematical model of roll motion, where nonlinear quadratic damping and nonlinear restoring based on the vessel restoring arm curve (GZ) have been considered. The structure of this model is the following

$$
\ddot{\phi}+2 \nu \omega_{0} \dot{\phi}+\beta \dot{\phi}|\dot{\phi}|+\omega_{0}{ }^{2} \frac{\overline{G Z}(\phi)}{\overline{G M}}=\omega_{0}{ }^{2} m_{\text {wave }}(t)
$$

where $\phi$ is the roll angle, $\nu$ and $\beta$ are the linear and quadratic roll damping coefficients, $\overline{G Z}(\phi)$ and $\overline{G M}$ are the righting lever curve and the metacentric height in still water, and $m_{\text {wave }}(t)$ is the non-dimensional wave excitation in irregular beam seas. The wave excitation has been modelled through the Absolute Roll Angle Model (Bulian and Francescutto, 2006) according to the following expression

$$
m_{\text {wave }}(t)=\sum_{i=1}^{n} \pi r\left(\omega_{i}\right) s\left(\omega_{i}\right) \cdot \cos \left(\omega_{i} t+\xi_{i}\right)
$$

where $\omega_{i}$ is the frequency of the $i$-th wave component, $r\left(\omega_{i}\right)$ is the effective wave slope coefficient, $s\left(\omega_{i}\right)$ is the wave steepness and $\xi_{i}$ is the phase.

This model (Equation 3) is derived from the classical linear model of uncoupled roll motion in waves (Bhattacharyya, 1978), and its detailed description can be found in Bulian and Francescutto (2004, 2006). In the first reference, it is applied to estimate the capsize probability of a small fishing vessel and a large passenger ship in irregular beam seas, while in the second 
it is used to analyze the operability and capsize probability of the same fishing vessel, in regular and irregular beam waves, as a function of its static stability parameters. In addition to these applications, this model has also been used for characterizing the roll motion of different types of multihull vessels in beam waves, comparing the results with scale model towing tank tests, with good results (Bulian and Francescutto, 2009).

Fishing vessels' operations usually take place at low speeds (while reeling in or recovering fishing gear, and even while processing catches) and in beam waves (as is the case of longliners). This brings about a high possibility of suffering stability failures or experiencing pure roll resonance characterized by large roll amplitudes due to the small roll damping at low speeds. Therefore, the present work focuses on the operational scenario characterized by zero forward speed and beam waves for its relevance and criticality. However, an extension to other headings and speed would be necessary to fully assess the performance of the methodology proposed in this paper.

The considered vessel is a medium sized stern trawler whose description and parameters are given in Section 4. To assess the effect of an external excitation on the resulting roll spectrum, three 1 hour simulations in beam waves were run using a Bretschneider spectrum, with peak periods that are under $\left(T_{p}=7 \mathrm{~s}\right)$, close to $\left(T_{p}=12.5 \mathrm{~s}\right)$ and over $\left(T_{p}=16 \mathrm{~s}\right)$ the roll natural period of the vessel $\left(T_{0}=11.16 \mathrm{~s}, \omega_{0}=0.563 \mathrm{rad} / \mathrm{s}\right)$. Regarding significant wave height, a constant value of $H_{s}=6 \mathrm{~m}$ has been selected, in order to ensure that the effect of waves is sufficiently evident on roll motion. For the sake of comparison, the results of the roll natural frequency estimation obtained from a roll decay test (where no external excitation was present), are also included. In order to estimate the roll natural frequency, the FFT has been applied to each roll time series to compute the roll spectrum, and the location of its maximum value has been taken as the natural frequency estimate for each time series.

Figures 1-3 show the roll time series in forced and unforced conditions, the corresponding roll spectra and the estimated roll natural frequency together with the frequency target value (shown by the black dotted line). As expected the roll spectrum obtained for the roll decay test (see Figure 3(d)) has a single peak, which coincides with the vessel roll damped frequency. However, the situation changes when wave excitation is taken into consideration.

When the wave peak frequency is close to the expected natural roll frequency (Figure 3(b)), or when it is smaller (Figure 3(c)), the resulting roll spectrum is not largely affected by the effect of waves. As it can be observed 


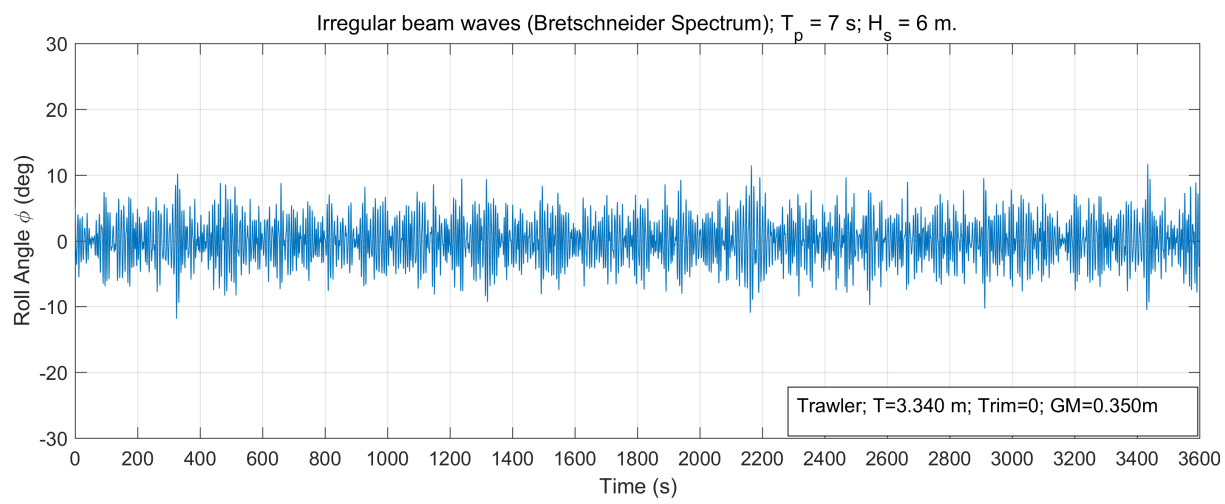

(a) $T_{p}=7 \mathrm{~s} ; H_{s}=6 \mathrm{~m}$.

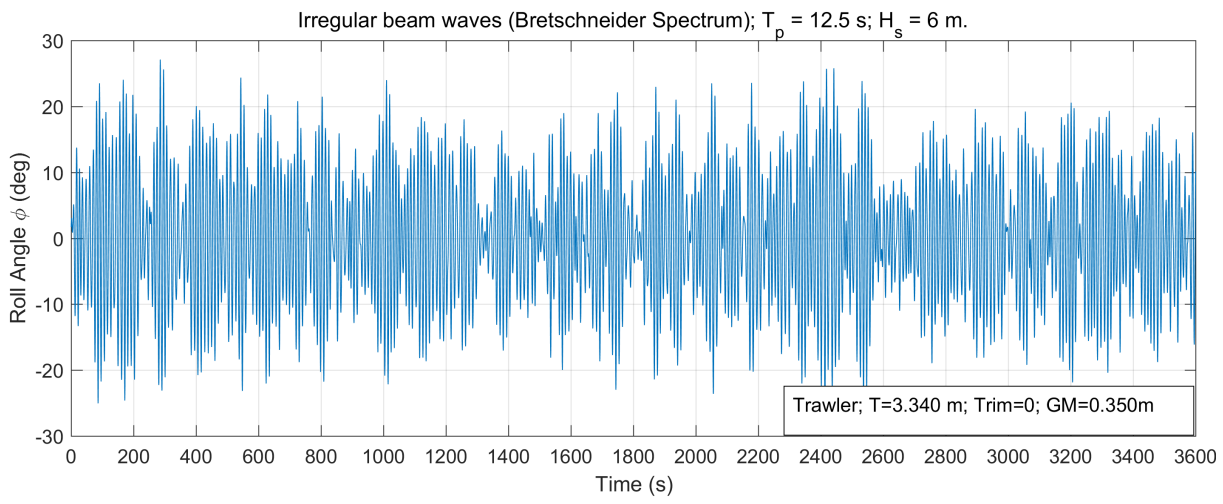

(b) $T_{p}=12.5 \mathrm{~s} ; H_{s}=6 \mathrm{~m}$.

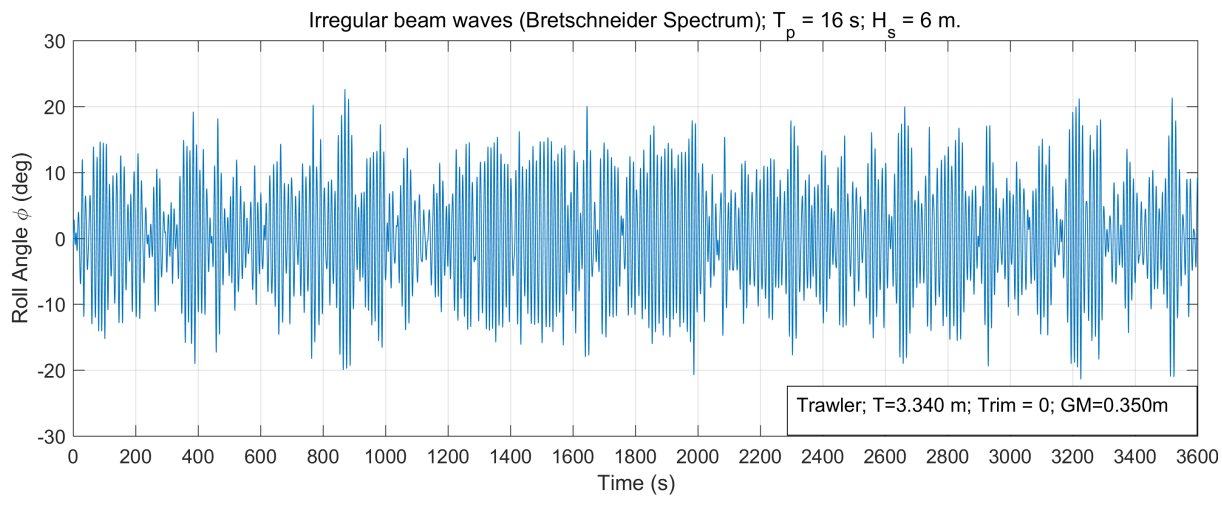

(c) $T_{p}=16 \mathrm{~s} ; H_{s}=6 \mathrm{~m}$.

Figure 1: Computed roll motion time series. Irregular waves. Bretschenider spectrum. 


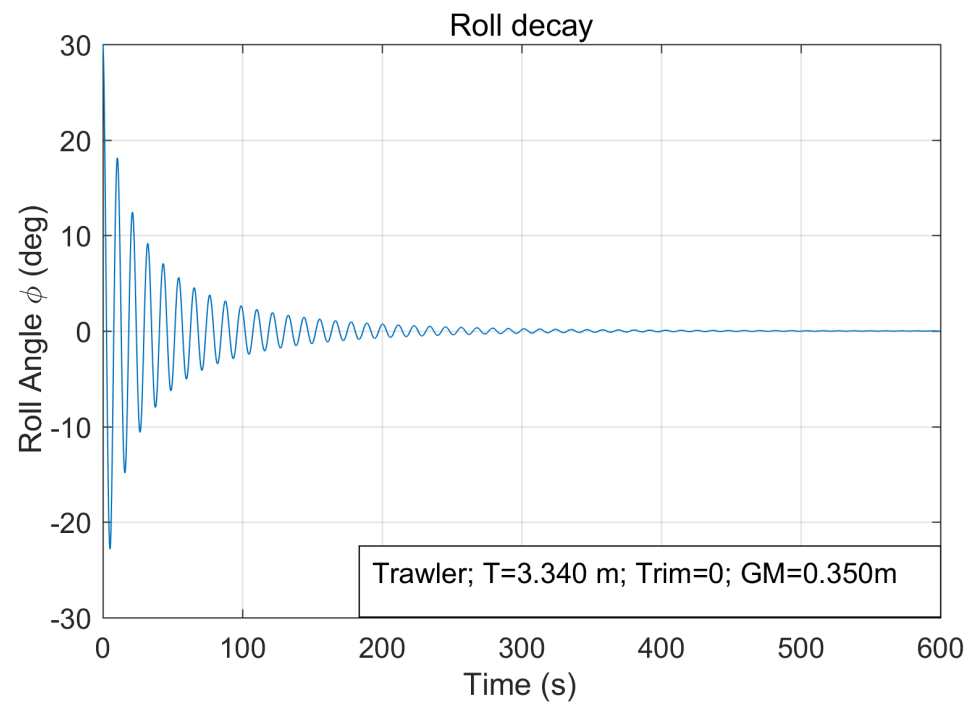

Figure 2: Computed roll motion time series. Roll decay. No external excitation.

in these figures, the peak frequencies of the roll spectra are very close to the natural roll frequency of the vessel, and the different wave components, specially those close to the peak of the wave spectrum, are of much less relevance than the roll motion component. This fact is due to the dynamic characteristics of the vessel roll motion, which tends to reduce the effects of the excitation that does not lead to roll oscillations near the natural frequency.

However, when the incident wave frequency is higher than the natural roll frequency (see Figure 3(a)), this behaviour changes dramatically and the resulting roll spectrum presents a scattered multi-peaked shape, which decreases the performance of the estimation. In this case, the peaks of the roll spectrum corresponding to the different wave frequency components, are much larger than those corresponding to the vessel roll natural frequency. As it can be seen, the estimated frequency value coincides with the wave peak frequency, which is quite far from the target value, and the vessel, in these conditions, is unable to cut down the effect of waves which lead to motions far from the natural frequency.

In addition to the influence of waves on the stability estimation, which was shown above, in a realistic seaway the vessel loading condition could largely change with time. This change can be generated by modifications in 


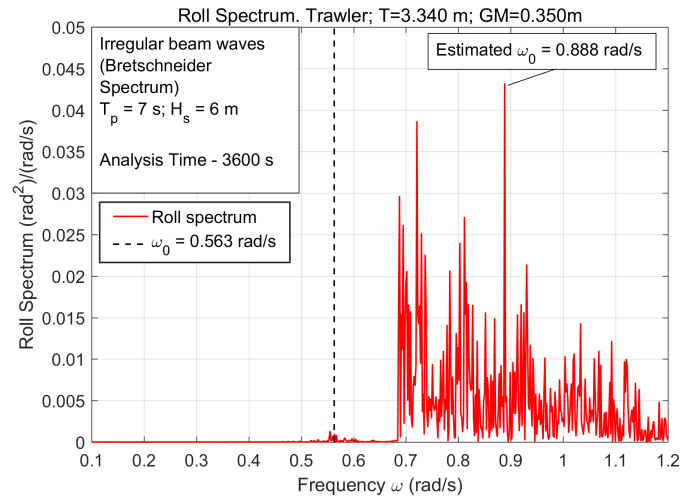

(a) Irregular waves. $T_{p}=7 \mathrm{~s} ; H_{s}=6 \mathrm{~m}$.

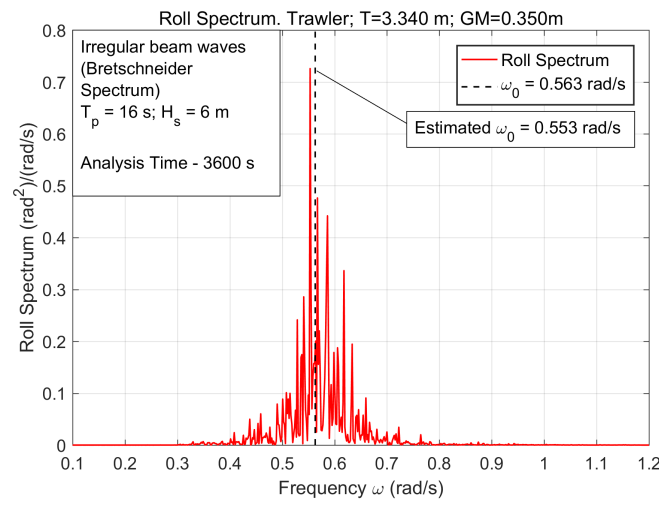

(c) Irregular waves. $T_{p}=16 \mathrm{~s} ; H_{s}=6 \mathrm{~m}$.

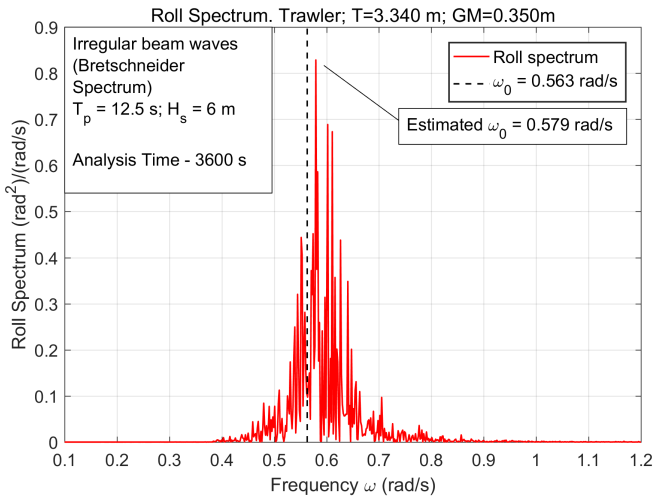

(b) Irregular waves. $T_{p}=12.5 \mathrm{~s} ; H_{s}=6 \mathrm{~m}$.

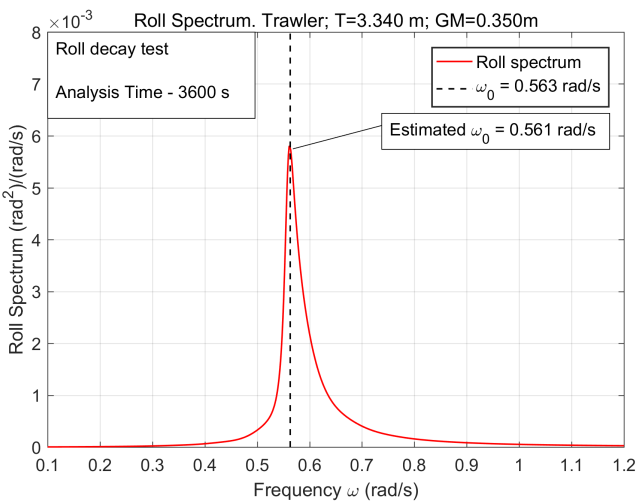

(d) Roll decay. No external excitation.

Figure 3: Computed roll spectra. 
the tank filling levels and especially in the amount of cargo, that can lead to quite fast and sudden stability changes. This issue would require to carry out the spectrum computations in shorter periods of time, thus decreasing the resolution of the FFT and subsequently the precision of the frequency estimation.

\subsection{Constraints on the roll natural frequency}

Finally, it should be said that, although during sailing the loading condition of the vessel may change in a sensible way, the resulting $G M$, and so the roll natural frequency should be, in reality, within more constrained limits than $0 \leq \omega_{o} \leq \infty$. This fact could help during the process of natural frequency estimation, as the range of variation is largely reduced, limiting $\omega_{0}$ to be in the interval $\omega_{0, \min } \leq \omega_{0} \leq \omega_{0, \max }$.

The maximum value of the roll natural frequency $\left(\omega_{0, \max }\right)$, corresponds to the maximum possible $G M$, of the vessel in all loading conditions, that if there is no other data available, could be estimated by considering that the vessel centre of gravity is on the base line. Thus, maximum GM would be equal to the maximum height of the metacenter $K M$, that could be easily obtained by analyzing the hydrostatical values corresponding to all drafts between lightship and summer draft. In the case under analysis, the maximum natural frequency would be $\omega_{0, \max }=2 \mathrm{rad} / \mathrm{s}$. However, in this work, as data from the stability booklet is available, $\omega_{0 \text {,max }}$ has been taken as the one corresponding to the largest GM condition from all of those described in this booklet, plus a $15 \%$ margin $\left(\omega_{0, \max }=0.925 \mathrm{rad} / \mathrm{s}\right)$.

Regarding the minimum roll natural frequency $\omega_{0, \min }$, it has to be taken into account that ship stability is not only limited by $G M$, and that in a real seaway, large roll angle stability is usually more relevant. Considering that according to SOLAS, marine engines could stop when static heel is over 15 degrees, it could be assumed that a vessel that acquires such an angle under the effect of moderate lateral winds, would be hardly able to sail. Thus, the minimum expected roll natural frequency $\left(\omega_{0, \min }\right)$ has been selected as the one corresponding to the minimum GM needed to keep heel angles under 15 degrees under the action of a lateral wind of approximately 30 knots. This lateral excitation, computed following the IMO Weather Criterion guidelines, corresponds to a heeling lever of $l_{\text {wind }}=0.03 \mathrm{~m}$. According to the vessel characteristics shown in Míguez González et al. (2017), the minimum expected natural roll frequency would then be $\omega_{0, \min }=0.3 \mathrm{rad} / \mathrm{s}$. These limits provide a conservative view of the whole range of possible natural roll frequencies. 
However, further work would be needed to obtain more realistic values from the real operation of the ships.

In conclusion, there are two outstanding problems for the accurate estimate of the vessel stability level during real operation. The first is to estimate the roll natural frequency (and then the metacentric height) by using only the vessel roll motion despite the effects of waves and other external excitation. The second is to ensure that the estimation is able to track sudden changes in roll natural frequency due to fast variations in the vessel loading condition.

\section{Condition monitoring system design}

A condition monitoring system able to address the aforementioned problems must be based on an estimation scheme that ideally is insensitive to wave motion and that is able to process measured data in (near) real-time.

Wave filters of different complexity have been proposed in connection with the design of autopilots and dynamic positioning systems to mitigate the effect of wave loads on vessel actuators (Fossen and Perez, 2009; Holzhiiter, 1992; Grimble et al., 1980; Sagatun and Fossen, 1996; Strand and Fossen, 1999). Despite their proven efficacy in attenuating wave frequency components on the measured signals, particularly when the wave peak frequency is known, complete insensitivity to wave motion cannot be achieved in reality. Further, the most powerful designs in terms of estimation accuracy are based on knowledge of a model of the vessel. This implies that regardless of the chosen estimation scheme the estimate of the roll natural frequency will always present a scatter around the true value due to residual wave induced motion, uncertainty in the vessel model parameters and possibly sensor noise.

To achieve a robust and portable stability monitoring system we propose a design that is vessel model-free and adopts probabilistic methods to discern among different operational conditions. In particular the monitoring system is developed as an integrated data-driven estimator-detector that features the following characteristics:

- real-time estimation of the roll natural frequency based solely on the measured roll angle;

- probabilistic model of the roll natural frequency;

- on-line continuous learning; 


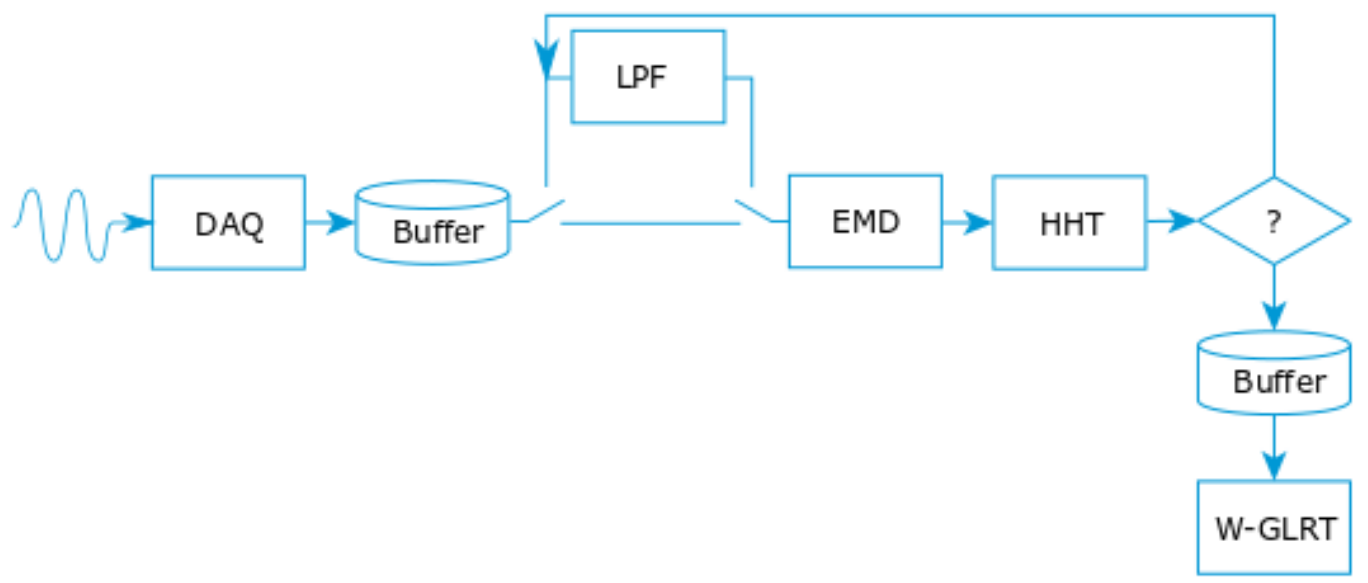

Figure 4: Architecture of the roll stability monitoring system.

- high sensitivity in discriminating across different loading/operational conditions;

- timely detection of unsafe operational conditions;

- low probability of false alarms;

- no need of external inputs from the crew.

Considering the previous characteristics, the current proposal differs in two main points from the previous work carried in (Míguez González et al., 2017; Santiago Caamaño et al., 2018b). On the one hand, this work was based on the sequential application of FFT to estimate the roll natural frequency and directly from this value the stability of the vessel. On the other hand, it lacked a system that provided additional information to the crew, thus relying only on the performance of the frequency estimates.

In the newly proposed methodology the combined EMD-HHT is used instead of the FFT to better discriminate the oscillatory modes contained in the roll motion, avoiding the tendency of the FFT to condensate information. In addition to this, the FFT needs a longer data batch to obtain accurate results, which in the case under analysis may lead to hiding stability failures that occur in very short time. Moreover, a detection system based on a probabilistic approach has been included to distinguish between safe and unsafe situations, increasing robustness of the generated stability guidance. 
Figure 4 illustrates the architecture of the proposed stability monitoring system. The main building blocks are

DAQ roll motion is measured through an inertial measurement unit or a vertical reference unit and stored in a buffer by the data acquisition (DAQ) system;

EMD the Empirical Mode Decomposition method (Dätig and Schlurmann, 2004; Gupta et al., 2014; Huang et al., 1998) is applied to decompose the measured roll motion into its main oscillatory modes;

HHT the Hilbert-Huang Transform is applied to each of the resulting oscillatory modes to estimate their modal frequency (Dätig and Schlurmann, 2004; Huang et al., 1998). Among all of these frequencies, the vessel roll natural frequency is selected;

W-GLRT the Weibull based Generalized Likelihood Ratio Test evaluates the current estimate of the roll natural frequency and decides if the vessel is operating in a safe loading conditions or not. The detector triggers an alarm if a safety threshold is crossed;

LPF Low-pass filtering of the measured roll motion is applied when the estimated roll natural frequency is close to the maximum roll natural frequency. When filtering occurs then the filtered signal is processed again through the EMD and HHT blocks.

In the following subsections each building block is described in detail.

\subsection{Data acquisition and storage}

The DAQ subsystem is responsible for the measuring of the vessel roll motion and storage in a buffer. To fulfill the real-time estimation requirement a proper buffer size needs to be determined. The choice has been made assuming a medium sized fishing vessel and by trading-off among the minimum data batch size to achieve a reliable estimate of the roll natural frequency, the maximum time to detect the transition towards an unsafe operational condition and the needed resolution to distinguish between different operational conditions. Following the same asumptions made in Míguez González et al. (2017), the time window length has been set to 3 minutes, which correspond to a buffer size of 3600 samples when sampling at $20 \mathrm{~Hz}$. 
In addition to this, a $75 \%$ of overlapping between consecutive measurements has been taken into account to increase the number of estimations done by time window.

\subsection{Empirical Mode Decomposition}

The Empirical Mode Decomposition (EMD) is an adaptive time domain technique that allows breaking down a multi-frequency signal into its main oscillatory components called Intrinsic Mode Functions (IMFs). The IMF is defined as a function with a time-varying frequency and amplitude and with equal number of extremes and zero-crossings, and whose envelopes are symmetric with respect to zero (Dätig and Schlurmann, 2004; Gupta et al., 2014; Huang et al., 1998).

The procedure to extract the IMFs is called sifting and it consists of connecting all the time series maxima with a cubic spline and all the minima with another one and obtaining the mean curve from both splines. If the difference between the time series and this mean curve satisfies the conditions of an IMF (equal number of extremes and zero-crossings and symmetry to zero), it is subtracted from the time series and the process is started again, until the result is a monotonic function. If the difference between the data and the mean curve is not an IMF, the sifting continues with the modified time series until an IMF is obtained or until one of the stopping criteria are fulfilled (Dätig and Schlurmann, 2004; Gupta et al., 2014; Huang et al., 1998). Algorithm 1 summarizes the sifting process.

The main objective of the EMD is decomposing the roll motion time series into its main oscillatory modes for the subsequent application of the Hilbert Spectral Analysis. After the application of the EMD the roll motion time series can be represented as

$$
\phi(t)=\sum_{i=1}^{N_{\mathrm{IMF}}} \operatorname{IMF}_{i}(t)+R(t), \quad t \in[\bar{t}-3 \min , \bar{t}]
$$

where $R(t)$ is a monotonic function, $N_{\mathrm{IMF}}$ is the total number of IMFs extracted from the measured roll motion and $\bar{t}$ is the current time.

In the case of a vessel rolling under the effect of an external excitation such as waves and wind, the extracted IMFs from the roll motion time series usually include the oscillatory modes due to this excitation, as well as the mode corresponding to the vessel natural frequency, sensor noise and other possible components. 


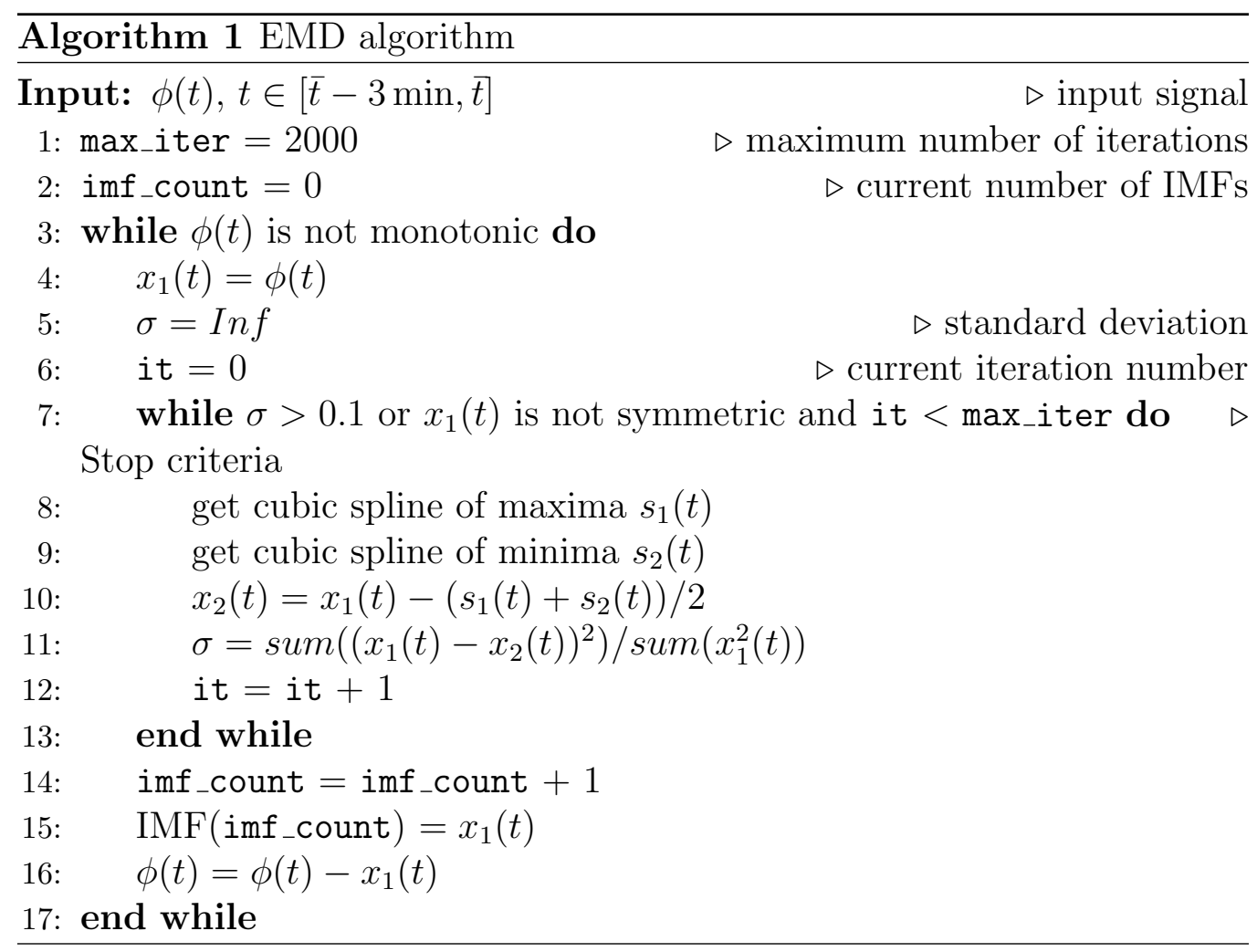




\subsection{Hilbert-Huang transform}

The Hilbert-Huang Transform (HHT) is a spectral analysis tool that transforms a real-valued function $x(t)$ into a complex one $z(t)$ that is timevariant. This new function can be written as (Dätig and Schlurmann, 2004; Huang et al., 1998)

$$
z(t)=x(t)+\mathrm{i} y(t)
$$

with $y(t)$ being the Hilbert-Huang Transform of $x(t)$.

HHT provides a representation of the signal in the time-frequency-energy paradigm. Another advantage of this method is that the new signal is strongly analytical, which means that it can be described in terms of instantaneous amplitude $(a(t))$, phase $(\theta(t))$ and frequency $(\omega(t))$. The definitions of each of these parameters are respectively (Dätig and Schlurmann, 2004; Huang et al., 1998)

$$
\begin{aligned}
& a(t) \triangleq \sqrt{x(t)^{2}+y(t)^{2}} \\
& \theta(t) \triangleq \arctan \left(\frac{y(t)}{x(t)}\right) \\
& \omega(t) \triangleq \frac{\mathrm{d} \theta(t)}{\mathrm{d} t}
\end{aligned}
$$

The Hilbert-Huang transform is applied to each IMF extracted from the roll motion in order to compute the associated instantaneous frequency, which in general is time varying. To obtain a constant frequency estimate for each IMF the mean instantaneous frequency is then computed according to (Xie and Wang, 2006)

$$
\hat{\omega}_{i}=\frac{\sum_{k=1}^{L_{\phi}} \omega_{i}(k) a_{i}^{2}(k)}{\sum_{k=1}^{L_{\phi}} a_{i}^{2}(k)}, \quad i=1, \ldots, N_{\mathrm{IMF}}
$$

where $L_{\phi}$ is the number of samples included in the time window $[\bar{t}-3 \mathrm{~min}, \bar{t}]$ given the sampling frequency $F_{s} ; \omega_{i}$ and $a_{i}$ are the instantaneous frequency and amplitude of the $i$-th IMF.

Once the mean instantaneous frequencies $\hat{\omega}_{j}$ of all IMFs are estimated, it is necessary to identify the roll natural frequency among them. The ordered

vector $\Omega_{\mathrm{IMF}}=\left[\hat{\omega}_{1}, \hat{\omega}_{2}, \ldots, \hat{\omega}_{N_{\mathrm{IMF}}}\right]^{\mathrm{T}}$ is created where $\hat{\omega}_{1}>\hat{\omega}_{2}>\ldots>\hat{\omega}_{N_{\mathrm{IMF}}}$ 
according to IMF extraction sequence of the EMD algorithm. Each element in $\Omega_{\mathrm{IMF}}$ is then assessed to be in the interval $\left[\omega_{0, \min }, \omega_{0, \max }\right]$ and the estimates that fall outside are discarded. At this point, it is assumed that the current roll natural frequency is the largest frequency, i.e. $\hat{\omega}_{0}=\max \Omega_{\mathrm{IMF}}$. This assumption is based on observations made on preliminary tests, which showed that most of the signal energy is associated with the first (and sometimes second) frequency in $\Omega_{\mathrm{IMF}}$. The rest of the components could therefore be neglected.

\subsection{Weibull GLR detector}

To take into account the inherent uncertainty of the estimation process, a statistical characterization of the estimated roll natural frequency is pursued by identifying the parametric probability distribution that best fit the estimates $\hat{\omega}_{0}$ across different loading conditions and sea states.

Figure 5 shows the probability plot of four distributions (logistic, tlocation scale, Weibull and double Weibull) fitted to a sequence of 90 estimates of roll natural frequency for loading conditions LC1, LC4 and LC6 (Table 2) and Sea State 1 (Table 3). As it can be seen in Table 2 LC1 is a safe condition, LC4 is the critical condition (the one with the minimum IMO required $G M$ ) and LC6 is a risky situation.

The probability plot shows that the Weibull distribution provides the most accurate fit to the estimates of the roll natural frequencies in all considered cases. The Weibull distribution describing $\hat{\omega}_{0}$ is given by

$$
\mathcal{W}\left(\hat{\omega}_{0}\right)=\frac{\kappa}{\lambda}\left(\frac{\hat{\omega}_{0}}{\lambda}\right)^{\kappa-1} \exp \left(-\left(\frac{\hat{\omega}_{0}}{\lambda}\right)^{\kappa}\right)
$$

where $\kappa$ is the shape parameter and $\lambda$ is the scale parameter. The probabilistic median of the Weibull distribution is a robust estimator of the roll natural frequency, i.e.

$$
\hat{\omega}_{0}=\lambda(\ln 2)^{\frac{1}{\kappa}}
$$

Further, it is function of both shape and scale parameters whose values change with variation in vessel loading condition, as shown in Fig. 5. This suggests that by designing a detector able to trail changes of $\kappa$ and $\lambda$ then it is possible to monitor variations in roll natural frequency, thereby evaluate the vessel stability condition in real-time.

The detection problem is set up as sequential hypothesis testing, where two competing hypotheses are evaluated for each batch of data. The null 

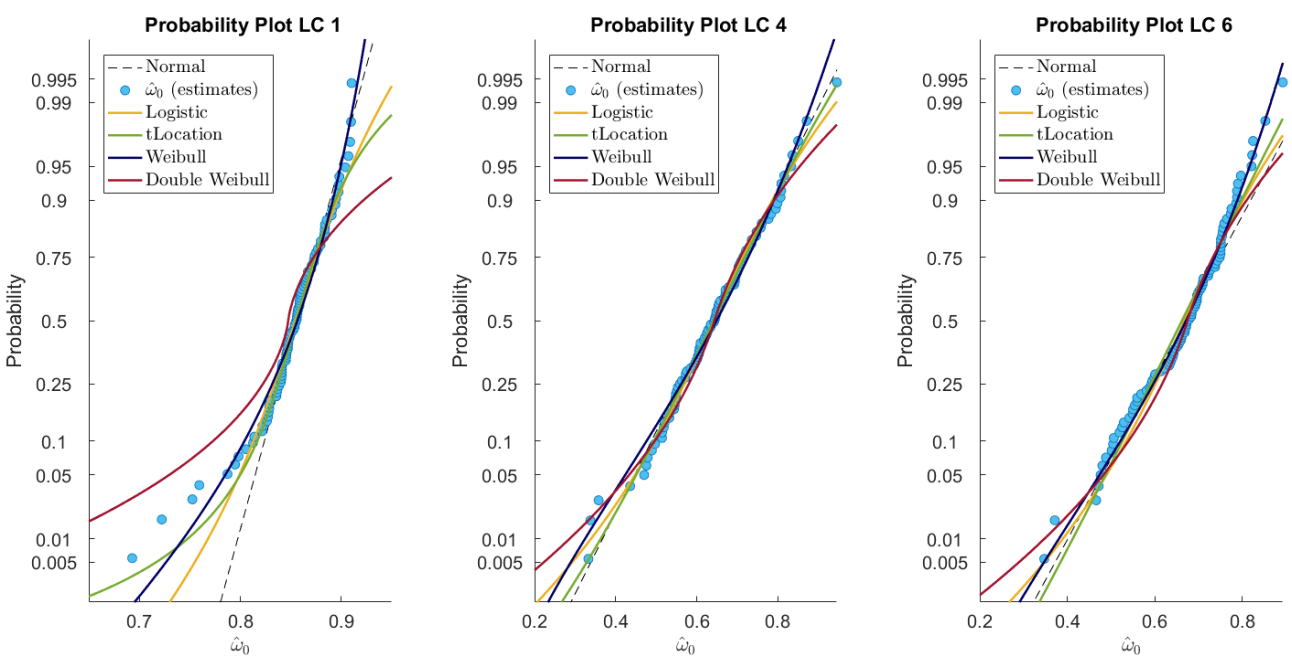

Figure 5: Probability plot of the roll natural frequency estimates for loading conditions LC1, LC4 and LC6, where $\omega_{0}=0.804 \mathrm{rad} / \mathrm{s}, \omega_{0}=0.563 \mathrm{rad} / \mathrm{s}$ and $\omega_{0}=0.514 \mathrm{rad} / \mathrm{s} \mathrm{respec-}$ tively, and Sea State 1. Logistic, t-location scale, Weibull and double Weibull distributions are fitted to the data.

hypothesis $\mathcal{H}_{0}$ represents the safe condition and the alternative hypothesis $\mathcal{H}_{1}$ corresponds to the unsafe condition, i.e.

$$
\begin{aligned}
& \mathcal{H}_{0}: \lambda_{0}(\ln 2)^{\frac{1}{\kappa_{0}}} \geq \omega_{0_{c}} \\
& \mathcal{H}_{1}: \hat{\lambda}_{1}(\ln 2)^{\frac{1}{\hat{\kappa}_{1}}}<\omega_{0_{c}}
\end{aligned}
$$

In ship stability, the safety of a loading condition depends on several parameters including $G M$, the heeling lever curve, the area under this curve, the freeboard, possible free surfaces, the ship-wave interaction, and few others. In this work the distinction between the two scenarios is done only using $G M$. The limiting $G M$ value between the safe and unsafe case has been taken as $0.350 \mathrm{~m}$, which is the minimum required $G M$ for fishing vessels according to IMO (International Maritime Organization, 2012). Therefore, using Equation (2), the critical roll natural frequency of the vessel corresponding to the $G M_{\min }=0.350 \mathrm{~m}$ is $\omega_{0_{c}}=0.563 \mathrm{rad} / \mathrm{s}$.

Since the median of the Weibull distribution is function of the shape and scale parameters, the detection problem in Equation (13) can be rewritten 
as a standard parameter test

$$
\begin{aligned}
& \mathcal{H}_{0}: \boldsymbol{\theta}=\boldsymbol{\theta}_{0} \\
& \mathcal{H}_{1}: \boldsymbol{\theta} \neq \boldsymbol{\theta}_{0}
\end{aligned}
$$

where $\boldsymbol{\theta}=[\lambda, \kappa]^{\mathrm{T}}$ is the vector of parameters of the Weibull distribution and $\boldsymbol{\theta}_{0}$ is its representation under the $\mathcal{H}_{0}$ hypothesis.

This corresponds to a composite hypothesis testing problem, since the alternative hypothesis $\mathcal{H}_{1}$ is not completely defined, as the parameters of the Weibull distribution which characterize this condition are unknown. In order to decide between the two competing hypotheses from a data set, the Generalized Likelihood Ratio Test (GLRT) is adopted. It is a statistical test based on the Neyman-Pearson theorem that maximizes the probability of detection for a desired probability of false alarms (Kay, 1998).

Let $\Omega_{0}=\left[\hat{\omega}_{0, k-N}, \ldots, \hat{\omega}_{0, k}\right]^{\mathrm{T}}$ the vector containing the $N$ latest estimates of roll natural frequency. Assuming that the elements of $\Omega_{0}$ are independent and identically distributed (i.i.d.), then its distribution reads

$$
\mathcal{W}\left(\boldsymbol{\Omega}_{0} ; \boldsymbol{\theta}\right)=\left(\frac{\kappa}{\lambda^{\kappa}}\right)^{N} \prod_{i=0}^{N-1}\left[\boldsymbol{\Omega}_{0, i}^{\kappa-1} \exp \left(-\left(\frac{\boldsymbol{\Omega}_{0, i}}{\lambda}\right)^{\kappa}\right)\right]
$$

The detector then decides that the current condition corresponds to $\mathcal{H}_{1}$ if:

$$
L_{G}\left(\boldsymbol{\Omega}_{0}\right)=\frac{\mathcal{W}\left(\boldsymbol{\Omega}_{0} ; \hat{\boldsymbol{\theta}}_{1}, \mathcal{H}_{1}\right)}{\mathcal{W}\left(\boldsymbol{\Omega}_{0} ; \boldsymbol{\theta}_{0}, \mathcal{H}_{0}\right)}>\gamma
$$

where $\hat{\boldsymbol{\theta}}_{1}$ is the maximum likelihood estimate (MLE) of the parameter vector $\boldsymbol{\theta}$ under the hypothesis $\mathcal{H}_{1}$ and $\gamma$ is the threshold for a given probability of false alarms.

The MLE $\hat{\boldsymbol{\theta}}_{1}$ is obtained maximizing $\mathcal{W}\left(\boldsymbol{\Omega}_{0} ; \boldsymbol{\theta}\right)$ under the hypothesis $\mathcal{H}_{1}$, that is

$$
\frac{\partial \ln \left(\mathcal{W}\left(\boldsymbol{\Omega}_{0} ; \hat{\boldsymbol{\theta}}_{1}, \mathcal{H}_{1}\right)\right)}{\partial \theta_{j}}=0
$$

Hence, the parameters $\hat{\lambda}_{1}$ and $\hat{\kappa}_{1}$ can be estimated as:

$$
\begin{gathered}
\hat{\lambda}_{1}=\left[\frac{1}{N} \sum_{i=0}^{N-1} \Omega_{0, i}^{\hat{\kappa}_{1}}\right]^{\frac{1}{\hat{\kappa}_{1}}} \\
\frac{1}{\hat{\kappa}_{1}}+\frac{1}{N} \sum_{i=0}^{N-1} \ln \left(\Omega_{0, i}\right)-\frac{\sum_{i=0}^{N-1} \Omega_{0, i}^{\hat{\kappa}_{1}} \ln \left(\Omega_{0, i}\right)}{\sum_{i=0}^{N-1} \Omega_{0, i}^{\hat{\kappa}_{1}}}=0
\end{gathered}
$$


The following explicit expression of the detector, can be derived by taking the natural logarithm of both sides of Equation (16)

$$
\ln \frac{\left(\frac{\hat{\kappa}_{1}}{\hat{\hat{\lambda}}_{1}^{\hat{\kappa}_{1}}}\right)^{N} \prod_{i=0}^{N-1}\left[\boldsymbol{\Omega}_{0, i}^{\hat{\kappa}_{1}-1} \exp \left(-\left(\frac{\sum_{i=0}^{N-1} \boldsymbol{\Omega}_{0, i}}{\hat{\lambda}_{1}}\right)^{\hat{\kappa}_{1}}\right)\right]}{\left(\frac{\kappa_{0}}{\lambda_{0}^{\kappa_{0}}}\right)^{N} \prod_{i=0}^{N-1}\left[\Omega_{0, i}^{\kappa_{0}-1} \exp \left(-\left(\frac{\sum_{i=0}^{N-1} \boldsymbol{\Omega}_{0, i}}{\lambda_{0}}\right)^{\kappa_{0}}\right)\right]}>\ln \gamma
$$

Simplifying the previous Equation (20), the GLRT becomes:

$$
\begin{array}{r}
N \ln \left(\frac{\hat{\kappa}_{1} \lambda_{0}^{\kappa_{0}}}{\kappa_{0} \hat{\lambda}_{1}^{\hat{\kappa}_{1}}}\right)+\left(\hat{\kappa}_{1}-\kappa_{0}\right) \sum_{i=0}^{N-1} \ln \left(\Omega_{0, i}\right)-\left(\frac{\sum_{i=0}^{N-1} \Omega_{0, i}}{\hat{\lambda}_{1}}\right)^{\hat{\kappa}_{1}} \\
+\left(\frac{\sum_{i=0}^{N-1} \Omega_{0, i}}{\lambda_{0}}\right)^{\kappa_{0}}>\gamma^{\prime}
\end{array}
$$

The threshold $\gamma^{\prime}=\ln (\gamma)$ has been selected to maximize the probability of detection, according to the Neyman-Pearson theorem (Kay, 1998)

$$
P_{F A}=\int_{\left\{\boldsymbol{\Omega}_{0}: L_{G}\left(\boldsymbol{\Omega}_{0}\right)>\gamma^{\prime}\right.} \mathcal{W}\left(\boldsymbol{\Omega}_{0} ; \theta_{0}, \mathcal{H}_{0}\right) \mathrm{d} x
$$

The detector raises an alarm only when the risky condition is met. To provide greater awareness to the crew about the current vessel stability condition an intuitive situation awareness system is designed. Its objective is to provide information to the skipper about how far the vessel is from the critical condition, which will help him to adopt corrective measures. The situation awareness system compares the actual robust estimate of the roll natural frequency $\left(\hat{\lambda}_{1}(\ln 2)^{\frac{1}{\hat{\kappa}_{1}}}\right)$ with the frequency $\omega_{0_{c}}$ identifying the critical situation. Thresholds are then established to determine the stability margin of the vessel. In order to simplify even more the information that the skipper is receiving, the situation awareness' output is color coded from dark green (safe situation) to red (very risky condition). The color related to each 
threshold has been defined the following way

$$
\begin{aligned}
& \frac{\hat{\lambda}_{1}(\ln (2))^{\frac{1}{\hat{\kappa}_{1}}}}{\omega_{0_{c}}} \geq 1.3 \rightarrow \text { dark green } \\
& 1.3>\frac{\hat{\lambda}_{1}(\ln (2))^{\frac{1}{\hat{\kappa}_{1}}}}{\omega_{0_{c}}} \geq 1.1 \rightarrow \text { green } \\
& 1.1>\frac{\hat{\lambda}_{1}(\ln (2))^{\frac{1}{\hat{\kappa}_{1}}}}{\omega_{0_{c}}} \geq 1.05 \rightarrow \text { yellow } \\
& 1.05>\frac{\hat{\lambda}_{1}(\ln (2))^{\frac{1}{\hat{\kappa}_{1}}}}{\omega_{0_{c}}} \geq 1 \rightarrow \text { orange } \\
& 1>\frac{\hat{\lambda}_{1}(\ln (2))^{\frac{1}{\hat{\kappa}_{1}}}}{\omega_{0_{c}}} \rightarrow \text { red }
\end{aligned}
$$

\subsection{Filtering}

In cases where $\omega_{0}$ is close to $\omega_{0 \max }$, the Weibull distribution is no longer a good fit to the estimates of roll natural frequency. This is due to the fact that the estimates are scattered around the true value, as it can be seen on the tails of the distribution. Following the process of identifying the roll natural frequency from the mean instantaneous frequencies, the values that are larger than $\omega_{0_{\max }}$ are neglected. That is, smaller values that do not correspond with the roll natural frequency are taken as estimates. This translates into a cutoff of the right tail of the Weibull distribution and the resulting probability distribution becomes bi-modal.

This fact can be appreciated in Figure 6, where the histograms of two different loading conditions of a fishing vessel under irregular waves are plotted. In Figure 6(a), the histogram of the roll natural frequency estimates corresponding to a loading condition that is far from the maximum expected frequency is displayed. As it can be seen, this histogram fits well with the Weibull probability distribution function. Figure 6(b) shows the histogram corresponding to a loading condition that is close to $\omega_{0_{\max }}$. As it was mentioned, it can be appreciated that the right tail is smaller and the distribution becomes bi-modal.

In order to correct this issue, a filtering process of the roll motion signal has been designed to reduce the dispersion in the estimates and to retain the validity of the Weibull distribution as statistical model of the roll natural frequencies estimates across all loading conditions. The employed filter is 
LC 3

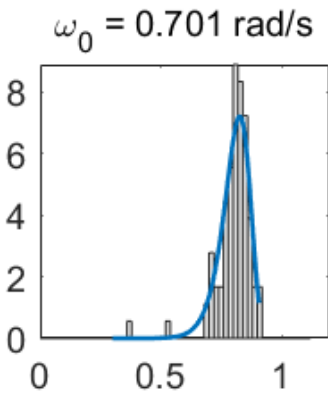

(a)
LC 1

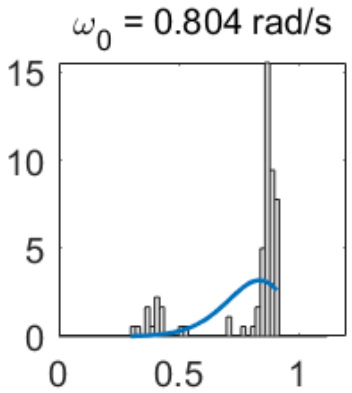

(b)
LC 1 with filter

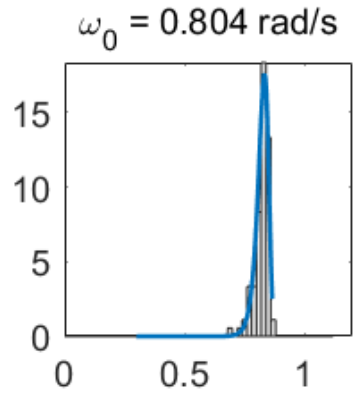

(c)

Figure 6: Histograms of $\hat{\omega}_{0}$. (a) Loading condition far from $\omega_{0_{\max }}$; (b) loading condition near $\omega_{0_{\max }}$ (b) without filtering; (c) loading condition near $\omega_{0_{\max }}$ after filtering. The blue line is the fitted Weibull distribution.

an eighth order lowpass Butterworth filter with zero phase distortion. This filtering process is applied to the stored 3 minutes batches of data, thus allowing the use of zero phase distortion techniques. The cutoff frequency is the highest roll natural frequency of the loading conditions described in the stability booklet $\left(\omega_{0 \max }\right)$.

Figure 6(c) shows the effect of filtering on the histogram of the estimated roll natural frequencies. By comparing with the histogram in Figure 6(b) it can be seen that filtering attenuates significantly the presence of outliers in the estimates and the Weibull distribution is again a good fit to the data.

As filtering is needed only in the described case, it is necessary to define its application limits. If the histogram in Figure 6(a) is taken into consideration, some dispersion can be observed. However, when the current loading condition natural roll frequency and its maximum expected value $\left(\omega_{0 \max }\right)$ are close to each other, the right tail of the resulting probability distribution starts to be cut and the dispersion in the results becomes larger. Therefore, the limit $\delta$ that determines the use of filtering or not is defined as

$$
\delta \triangleq \omega_{0 \max }-\frac{\sigma_{\hat{\omega}_{0}}}{2}
$$

where $\sigma_{\hat{\omega}_{0}}$ is the variance of the roll natural frequency estimates.

In conclusion, filtering of the measured roll motion is applied when the median of the Weibull distribution is greater than $\delta$. In this case, the EMD and HHT have to be applied again to the filtered signal to obtain $\hat{\omega}_{0}$. 
Table 1: Test vessel: main characteristics.

\begin{tabular}{lc}
\hline Overall Length & $34.50 \mathrm{~m}$ \\
Beam & $8.00 \mathrm{~m}$ \\
Depth & $3.65 \mathrm{~m}$ \\
Draft & $3.34 \mathrm{~m}$ \\
Hull Volume & $448 \mathrm{~m}^{3}$ \\
Linear Roll Damping Coefficient $(\nu)$ & 0.0187 \\
Quadratic Roll Damping Coefficient $(\beta)$ & $0.03931 / \mathrm{rad}$ \\
Minimum roll natural frequency $\left(\omega_{0 \mathrm{~min}}\right)$ & $0.300 \mathrm{rad} / \mathrm{s}$ \\
Maximum roll natural frequency $\left(\omega_{0 \mathrm{max}}\right)$ & $0.925 \mathrm{rad} / \mathrm{s}$ \\
\hline
\end{tabular}

\section{Test and validation}

\subsection{Fishing vessel model}

To evaluate the performance and sensitivity of the monitoring system, a set of different loading conditions have been analyzed and compared. Furthermore, the impact of the sea state on the performance of the proposed methodology has also been evaluated by comparing the results obtained in different sea states.

Roll motion time series have been obtained by using the 1 degree-offreedom nonlinear mathematical model described in Section 2. This model computes the vessel roll motion under the excitation of beam irregular seas at zero forward speed.

The vessel used to carry out this study is a mid-sized stern trawler, typical from the Spanish fishing fleet, which has been already taken as a test vessel in other scientific works (Míguez González et al., 2017; Míguez González and Bulian, 2018). Table 1 shows the main dimensions and characteristics of the vessel, together with roll damping coefficients obtained from roll decay tests of a scale model (Míguez González and Bulian, 2018).

The vessel hull form is shown in Figure 7. Figure 8 illustrates the vessel righting lever curve for the loading condition with minimum required $G M$ (LC4 in Table 2). The effective wave slope coefficient needed for the computation of the wave exciting moment $m_{\text {wave }}(t)$ is shown in Figure 9 , which has been obtained by using the linear hydrodynamics alternative as shown in Míguez González and Bulian (2018).

In order to verify how the detection system performs and how fast it recognizes possible changes in vessel stability, a set of 6 loading conditions 


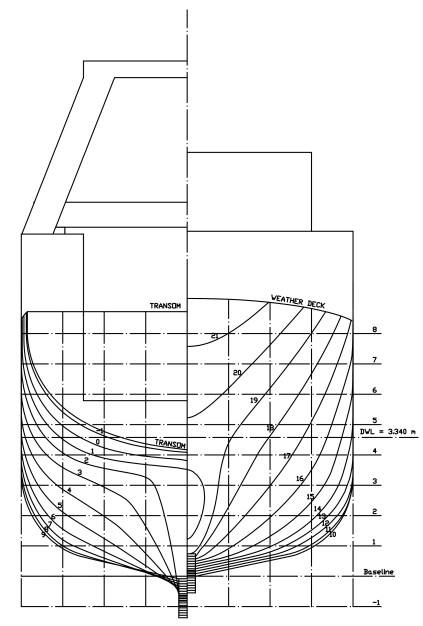

Figure 7: Hull form of the mid-sized sern trawler.

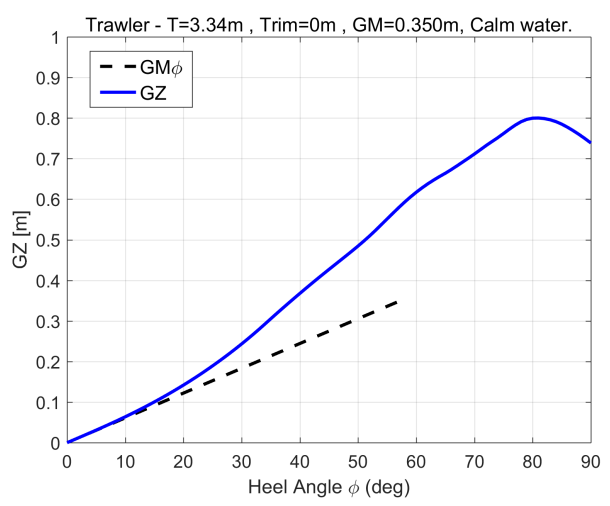

Figure 8: GZ curve in calm water.

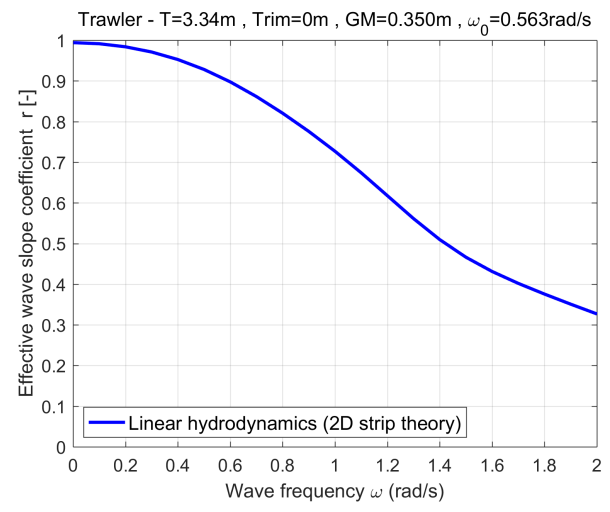

Figure 9: Effective wave slope coefficient.

were defined. LC 1 and LC 2 are two of the mandatory loading conditions contained in the stability booklet, being respectively "Departure from the fishing grounds with full catch and fishing gear, $35 \%$ of fuel and stores and no ice" and "Arrival at home port with full catch and fishing gear, 10\% of fuel and stores and no ice". In addition, LC 1 represents the loading condition with the expected maximum roll natural frequency (corresponding to the highest $G M$ ). LC 4 is the critical loading condition (the one with the minimum required metacentric height of $G M=0.350 \mathrm{~m}$ ) and LC 3 is an intermediate situation. All of them are under the $H_{0}$ hypothesis.

LC5 and LC6 loading conditions belong to the alternative hypothesis, 
Table 2: Loading condition parameters of the tested vessel.

\begin{tabular}{lcccccc}
\hline Parameter & $L C 1$ & $L C 2$ & $L C 3$ & $L C 4$ & $L C 5$ & $L C 6$ \\
\hline$\Delta(\mathrm{t})$ & 489 & 465 & 489 & 448 & 448 & 448 \\
$T(\mathrm{~m})$ & 3.295 & 3.199 & 3.484 & 3.340 & 3.294 & 3.294 \\
$G M(\mathrm{~m})$ & 0.659 & 0.661 & 0.501 & 0.350 & 0.331 & 0.291 \\
$\omega_{0}(\mathrm{rad} / \mathrm{s})$ & 0.804 & 0.798 & 0.701 & 0.563 & 0.548 & 0.514 \\
$k_{x x}$ & 0.395 & 0.399 & 0.395 & 0.411 & 0.411 & 0.411 \\
\hline
\end{tabular}

that is, they are supposed to be unsafe situations where $G M$ values are below the minimum required value. Due to the fact that these conditions do not fulfill the minimum stability requirements of the IMO for fishing vessels (International Maritime Organization, 2012), they are not contained in the compulsory stability booklet, and have been defined by the authors. The main objective of these last two loading conditions is to evaluate if the detector is able to identify them as dangerous situations and trigger the alarm. The main parameters of these loading conditions are shown in Table 2. In order to set up the model, righting lever curves in still water for the six loading conditions have also been computed by using a naval architecture software.

\subsection{Test conditions}

Environmental conditions were selected considering the possible influence of the wave encounter frequency in the estimation of the roll natural frequency. The range of tested waves covers all the relevant scenarios, that is, from a peak encounter frequency lower than the critical one, to a peak encounter frequency greater than the expected maximum. Waves have been generated using a Bretschneider spectrum (International Towing Tank Conference, 2002), considering the significant wave height $\left(H_{s}\right)$, peak frequency $\left(\omega_{\omega}\right)$ and wave steepness $\left(S_{\omega}\right)$ summarized in Table 3.

\subsection{Tuning of the condition monitoring system}

To theoretically determine the threshold $\gamma^{\prime}$ of the detector the components of vector $\Omega_{0}$ have to be i.i.d. (independent and identically distributed). If this condition is not met, the performance of the detector cannot be guaranteed from a theoretical standpoint and empirical methods for tuning should be pursued. Figure 10 shows the auto-correlation function of $\Omega_{0}$ under the null 
Table 3: Tested wave parameters.

\begin{tabular}{cccc}
\hline Sea State & $H_{s}(\mathrm{~m})$ & $\omega_{\omega}(\mathrm{rad} / \mathrm{s})$ & $S_{\omega}$ \\
\hline 1 & 12.810 & 0.491 & $1 / 20$ \\
2 & 9.720 & 0.563 & $1 / 20$ \\
3 & 7.080 & 0.660 & $1 / 20$ \\
4 & 4.830 & 0.798 & $1 / 20$ \\
5 & 3.030 & 1.008 & $1 / 20$ \\
6 & 1.650 & 1.369 & $1 / 20$ \\
7 & 8.520 & 0.491 & $1 / 30$ \\
8 & 6.480 & 0.563 & $1 / 30$ \\
9 & 4.710 & 0.660 & $1 / 30$ \\
10 & 3.240 & 0.798 & $1 / 30$ \\
11 & 2.010 & 1.008 & $1 / 30$ \\
12 & 1.320 & 1.369 & $1 / 30$ \\
13 & 2.550 & 0.491 & $1 / 100$ \\
14 & 1.950 & 0.563 & $1 / 100$ \\
15 & 1.410 & 0.660 & $1 / 100$ \\
16 & 0.960 & 0.798 & $1 / 100$ \\
17 & 0.600 & 1.008 & $1 / 100$ \\
18 & 0.330 & 1.369 & $1 / 100$ \\
\hline
\end{tabular}




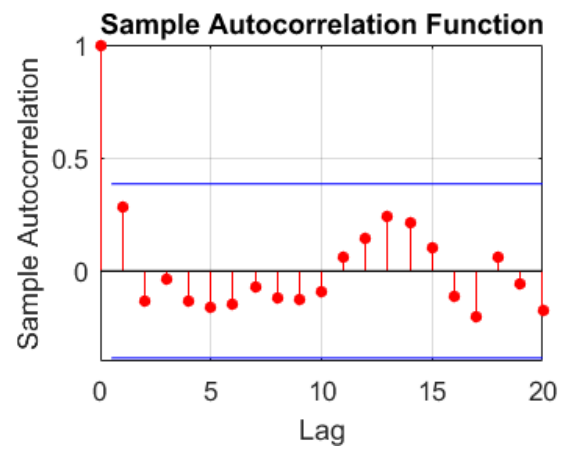

Figure 10: Auto-correlation function of the driving signal.

hypothesis. It can be seen that the data are uncorrelated and therefore the i.i.d. conditioned is fulfilled. Furthermore, this condition is also met when filtering is needed.

The threshold for a desired false alarm probability $\left(P_{F A}\right)$ is obtained from

$$
\begin{aligned}
1-P_{F A} & =1-\exp \left(-\left(\frac{\gamma^{\prime}}{\lambda_{0}}\right)^{\kappa_{0}}\right) \\
\gamma^{\prime} & =\lambda_{0}\left(-\ln \left(P_{F A}\right)\right)^{\frac{1}{\kappa_{0}}}
\end{aligned}
$$

For the hypothesis $\mathcal{H}_{0}$ shown in Figure 11, a false alarm probability of $P_{F A}=0.0000001$ is obtained for a threshold $\gamma^{\prime}=0.8407$.

As explained in Section 3.4, the W-GLRT is based on the assumption that the hypothesis $\mathcal{H}_{0}$ is already known, which is not usually true. In fact, it is supposed that when the vessel leaves port and the monitoring system is started, the loading condition should have changed from the last time the system was used.

However, since a key feature of the monitoring system is the ability to operate automatically without inputs from the crew, an estimation of the parameter vector $\boldsymbol{\theta}_{0}$ is done each time the system starts running. So, two different operation stages of the system can be distinguished.

Estimation stage This stage takes places immediately after the system is started, and ideally when the vessel is leaving port in protected waters. In this phase, during a time window $T_{e s t}$, the system is only collecting roll natural frequency estimates. Once this time is over, the parameter 
vector $\boldsymbol{\theta}_{0}$ is calculated according to Equations (18)-(19). If the probabilistic median of the Weibull distribution $\left(\lambda_{0}(\ln 2)^{\frac{1}{\kappa_{0}}}\right)$ is lower than $\omega_{0_{c}}$, an alarm is raised and a message should be displayed to the skipper to inform him about too low metacentric height for sailing. Conversely, $\boldsymbol{\theta}_{0}$ is stored as the reference value to be used during sailing.

In this paper, a $T_{e s t}=20 \mathrm{~min}$ is proposed, as during this period a large enough number of roll natural frequency estimates can be made. Furthermore, during this time the vessel is leaving the port, and considering that she is not facing a rough weather, the risk of suffering stability issues remains lower than in open seas.

Detection stage During this stage, the system is fully operational. $H_{0}$ is already known and consequently $H_{1}$ can be evaluated and compared against it, following the procedure described in Section 3.4. As it was previously done with $\boldsymbol{\theta}_{0}$, another time window $\left(T_{d e t}\right)$ is necessary to make the estimation of $\hat{\boldsymbol{\theta}}_{1}$. During this stage, ensuring the requirement of real-time is crucial. So, a detection time window of $T_{d e t}=5$ min has been selected, together with a $40 \%$ of overlapping between consecutive measurements. This leads to the GLRT releasing a new value every 3 minutes, thus fulfilling the real-time requirement described in Subsection 3.1 and providing enough roll natural frequency estimates to perform the MLE.

\subsection{Evaluation of monitoring performance}

The roll motion time series, corresponding to each of the sea states, have been obtained by stitching together those of the 6 loading conditions, in order from the safest to the most risky one, to simulate long record history (6.75 $\mathrm{h})$. Then, the estimation of the roll natural frequency was done and, at the same time, the GLRT detector was applied. Thus, the performance of the frequency estimation subsystem (EMD and HHT modules) and the detection subsystem ( $\mathcal{W}$-GLRT) can be analyzed. To address in detail the impact of the wave encounter frequency in the estimation and detection performance, two different wave situations are used as study cases. In addition, the complete results for all the sea states contained in Table 3 are shown in Table 6 .

Figure 11 is based on Sea State 7, Table 3, and it shows the roll motion time series, the roll natural frequency estimates, the output of the detector and the output of the awareness alarm in a case in which the wave encounter frequency is below the expected maximum roll natural frequency. As it can 


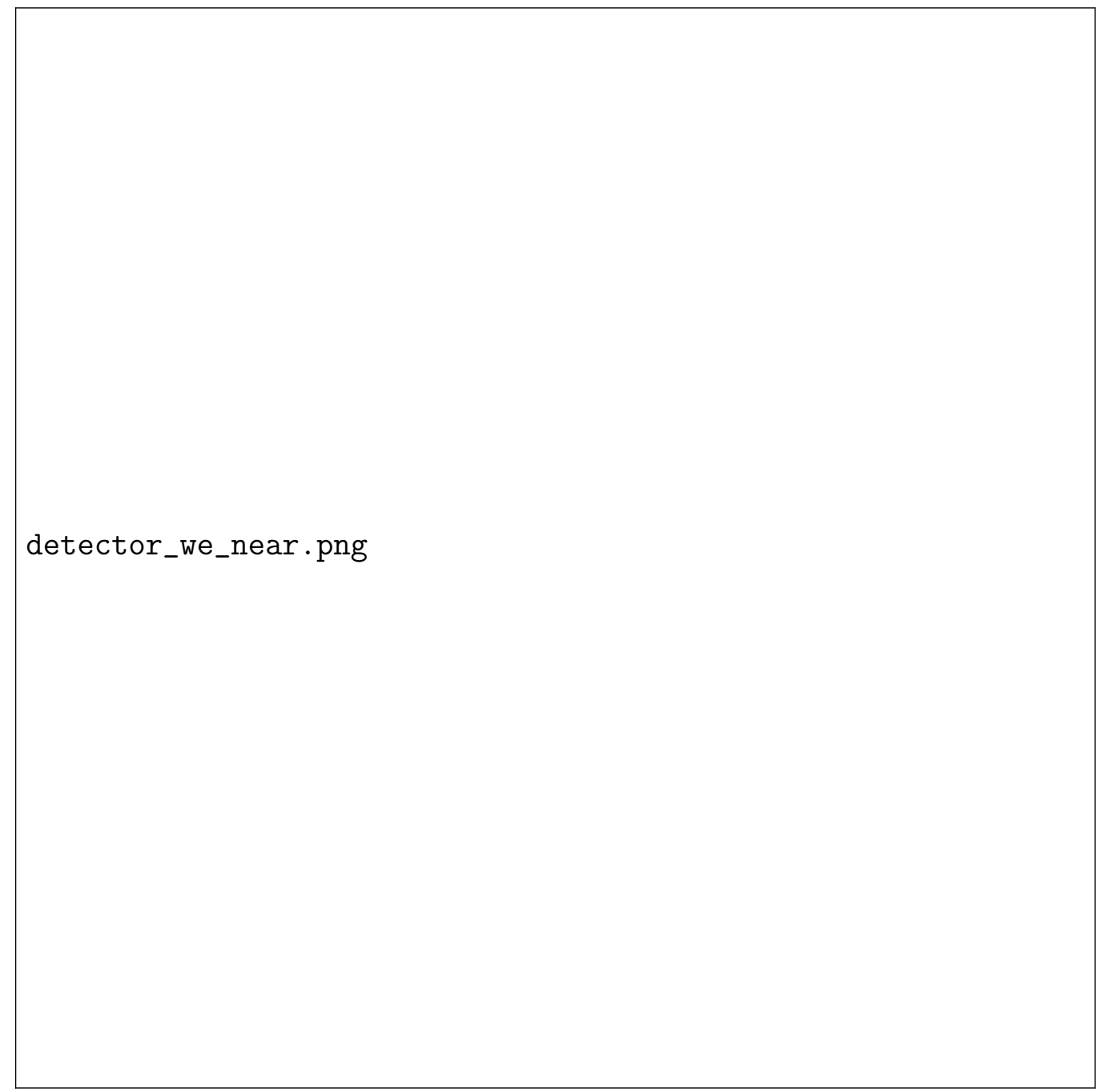

Figure 11: Top to bottom. Roll motion time series, natural roll frequency estimations, output of the detector and output of the awareness alarm in irregular waves. Sea State 7. $H_{s}=8.520 \mathrm{~m}, \omega_{w}=0.491 \mathrm{rad} / \mathrm{s}, S_{w}=1 / 30$. 
be seen the roll amplitude is large during the whole simulation. This is mainly due to the high significant wave height. Regarding the performance of the frequency estimation subsystem, some dispersion around the natural frequency of each loading condition can be observed. This dispersion becomes larger when the value of the natural frequency is smaller. The $\mathcal{W}$-GLRT is quite sensitive and all loading conditions could be perfectly distinguished without delay looking at its output. Its performance is also very good, as all loading conditions are correctly classified and no false alarms appear or missdetections take place. It could be also appreciated that the detector confuses the critical loading condition with a situation of danger, and subsequently triggers the alarm. Nevertheless, looking at the output of the awareness alarm, the critical condition could be clearly differentiated. In fact, the perception of risk is increasing as advancing through the loading conditions. Then it could be concluded that the awareness alarm performs adequately, although in some circumstances more than one color appears, which is due to the dispersion of the estimates.

Figure 12 is based on Sea State 17 and it illustrates again the roll motion time series, the roll natural frequency estimates, the output of the detector and the output of the awareness alarm but for a sea state in which the wave encounter frequency is greater than the maximum roll natural frequency. In this case, the roll amplitude decreases considerably, as wave height is quite small. The dispersion in the roll natural frequency estimates increases, specially in the loading conditions with the smallest rolling amplitudes (LC4, LC5 and LC6). Nonetheless, the performance of the detector is still quite good, adequately identifying safe and unsafe conditions. No false alarms arise and no miss-detections take place. However, the awareness alarm is not capable of correctly recognizing the changes in loading conditions, and it is only able to differentiate between safe and dangerous situations. As aforementioned, the complete results for all of the sea states are summarized in Table 4 and Table 6.

The performance of the frequency estimation subsystem has been evaluated by computing the median $\left(M e d_{\hat{\omega}_{0}}\right)$, the standard deviation $\left(\sigma_{\hat{\omega}_{0}}\right)$ and the 95th and 5th percentiles $\left(P_{95_{\hat{\omega}_{0}}}\right.$ and $\left.P_{5_{\hat{\omega}_{0}}}\right)$ of the roll natural frequency estimates, which have been included in Table 6 . The frequency estimation subsystem well performs except in those situations where the wave frequency is greater than the expected roll natural frequency and roll motions are of small amplitude. In these cases the combined EMD-HHT module largely overestimates the roll natural frequency of the vessel, which results in a 

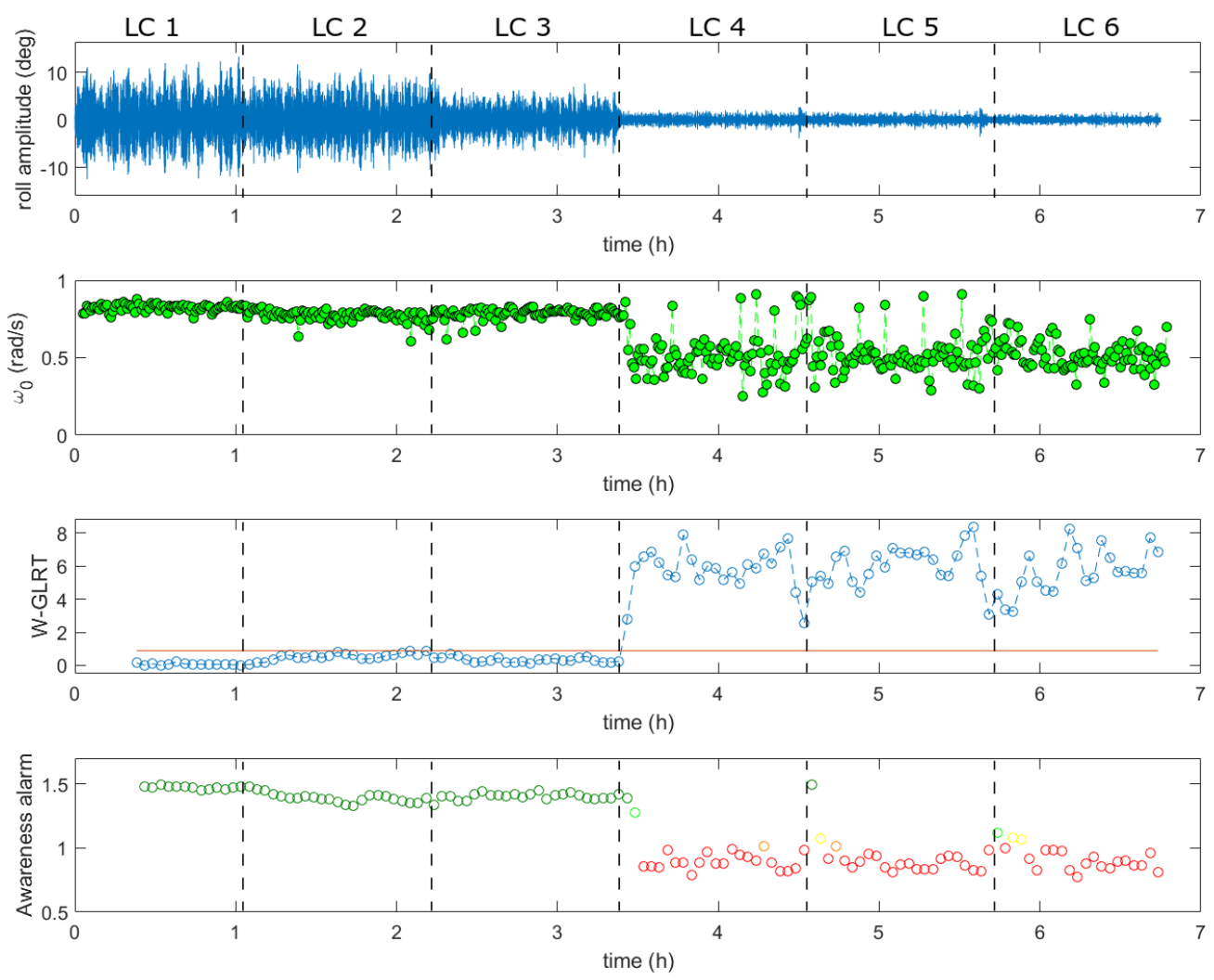

Figure 12: Top to bottom. Roll motion time series, natural roll frequency estimations, output of the detector and output of the awareness alarm in irregular waves. Sea State 17. $H_{s}=0.600 \mathrm{~m}, \omega_{e}=1.008 \mathrm{rad} / \mathrm{s}, S_{w}=1 / 100$. 
degradation of the performance of the detector and the awareness alarm. In order to illustrate this fact, Figure 13 has been included, where the median and the standard deviation corresponding to all loading conditions and sea states from 1 to 6 (those with a wave steepness of $S_{\omega}=1 / 20$ ) are shown. In this figure, it can be observed that in Sea State 4 and Sea State 6, the estimated natural roll frequency is largely over the target value for the conditions with a lower GM (LC4, LC5 and LC6), while in the other load cases and sea states, obtained results are much closer to the corresponding target values. This situation also takes place in Sea States 10, 12, 16 and 18. In these cases, wave height is very low, vessel roll motions are also of very small amplitude (similarly to the time series displayed in Figure 12) and, in addition, wave frequency is very far over the expected vessel natural roll frequency. This phenomenon is the same as the one represented in Figure 3 (a) .

In order to analyze the performance of the detector $(P D)$, the total number of true detections $(T D)$ and the total number of false detections $(F D)$ have been obtained, and are included in Table 4 . These values represent the number of times that the detector is able to adequately recognize if the loading condition under consideration is safe or not $(T D)$ and those in which it is not $(F D)$. From the obtained results, it could be observed that in the two loading conditions with better stability (LC1 and LC2), the detector performs very well in all sea states, and no false detections are made; results in LC3, although some false detections take place in Sea State 8, are still quite good. As the level of stability goes under the critical condition (LC4), the performance of the detector starts decreasing, but it still works fine in most of the sea states. However, in Sea State 6, Sea State 12 and Sea State 18, a very large number of false detections takes place in LC5 and LC6. Under these circumstances, as it was previously described, the EMD+HHT is hardly able to estimate the natural roll frequency which is why the performance of the detector gets worse. Despite of this fact, the detector is still able to distinguish between safe and not safe situations with a good accuracy even in situations in which the accuracy of the EMD+HHT is not extremely good.

In the case of the critical condition (LC4), the detector is unable to classify the loading condition as safe in most of the Sea States. However, and due to the fact that this situations represents the frontier between safe and unsafe, the obtained results could be acceptable as they represent a slightly conservative approach.

As a summary of the performance of the detector, the confusion matrix 


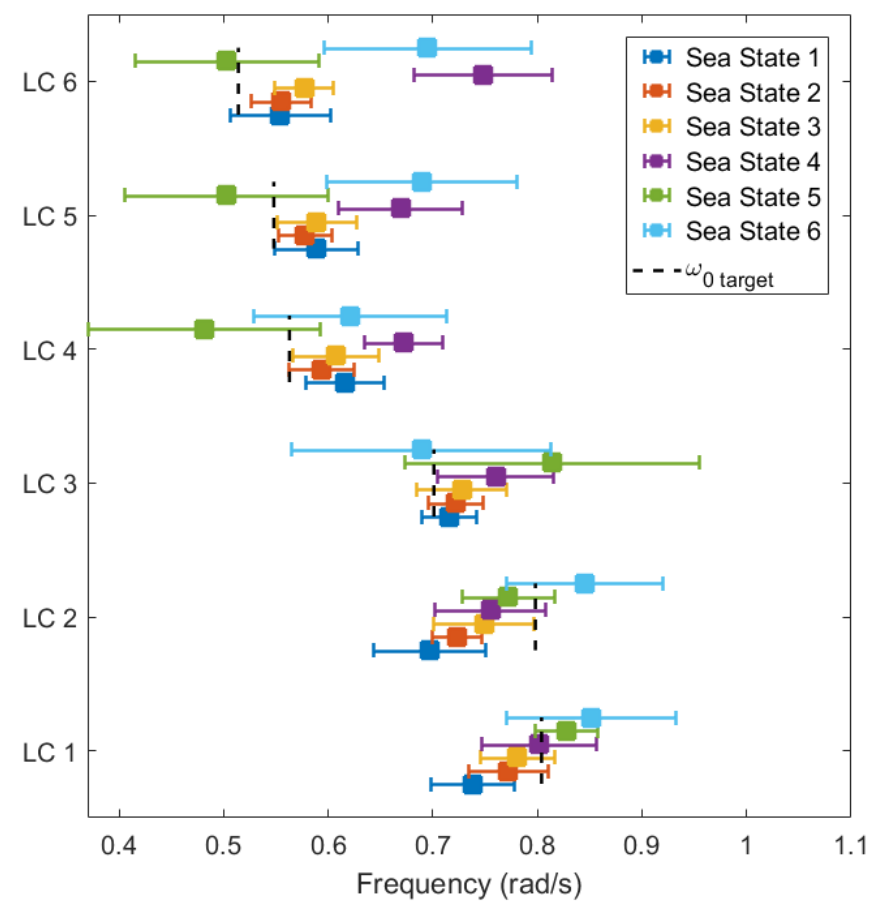

Figure 13: Median and standard deviation of natural roll frequency estimations. All load cases. Sea States 1 to 6.

is shown in Table 5 .

\subsection{Discussion}

From the presented results, it can be concluded that the proposed vessel's stability monitoring system displays a good-to-very good performance. It is capable of detecting potential risky situations and also of giving some information about how far the current condition from the safety limit is.

The combined EMD-HHT module can estimate the roll natural frequency of the vessel with an acceptable degree of dispersion. However, there is a number of sea states and loading conditions, which correspond to those with a lower level of stability and a wave peak frequency far from the expected roll natural frequency, where the module's performance decreases in comparison to the other situations.

This behaviour could be explained due to some of the fundamental assumptions which have been taken while developing the method. In order 
Table 4: Summary of results of the $\mathcal{W}$-GLRT detector.

\begin{tabular}{ccccccccccccc}
\hline \multirow{2}{*}{$\begin{array}{c}\text { Sea } \\
\text { State }\end{array}$} & \multicolumn{2}{c}{ TC 1} & \multicolumn{2}{c}{ LC 2} & \multicolumn{2}{c}{ LC 3} & \multicolumn{2}{c}{ LC 4} & \multicolumn{2}{c}{ LC 5} & \multicolumn{2}{c}{ LC 6} \\
& $T D$ & $F D$ & $T D$ & $F D$ & $T D$ & $F D$ & $T D$ & $F D$ & $T D$ & $F D$ & $T D$ & $F D$ \\
\hline 1 & 16 & 0 & 23 & 0 & 23 & 0 & 13 & 10 & 23 & 0 & 21 & 0 \\
2 & 16 & 0 & 23 & 0 & 23 & 0 & 1 & 22 & 23 & 0 & 21 & 0 \\
3 & 16 & 0 & 23 & 0 & 23 & 0 & 0 & 23 & 23 & 0 & 21 & 0 \\
4 & 16 & 0 & 21 & 2 & 23 & 0 & 2 & 21 & 18 & 5 & 5 & 16 \\
5 & 16 & 0 & 23 & 0 & 20 & 3 & 0 & 23 & 23 & 0 & 21 & 0 \\
6 & 16 & 0 & 23 & 0 & 23 & 0 & 23 & 0 & 0 & 23 & 0 & 21 \\
7 & 16 & 0 & 23 & 0 & 23 & 0 & 0 & 23 & 23 & 0 & 21 & 0 \\
8 & 16 & 0 & 23 & 0 & 1 & 22 & 0 & 23 & 23 & 0 & 21 & 0 \\
9 & 16 & 0 & 23 & 0 & 23 & 0 & 1 & 22 & 22 & 1 & 21 & 0 \\
10 & 16 & 0 & 23 & 0 & 15 & 8 & 0 & 23 & 23 & 0 & 10 & 11 \\
11 & 16 & 0 & 23 & 0 & 23 & 0 & 0 & 23 & 23 & 0 & 21 & 0 \\
12 & 16 & 0 & 23 & 0 & 18 & 5 & 17 & 6 & 5 & 18 & 3 & 18 \\
13 & 16 & 0 & 23 & 0 & 23 & 0 & 0 & 23 & 23 & 0 & 21 & 0 \\
14 & 16 & 0 & 23 & 0 & 23 & 0 & 1 & 22 & 23 & 0 & 21 & 0 \\
15 & 16 & 0 & 23 & 0 & 23 & 0 & 0 & 23 & 23 & 0 & 21 & 0 \\
16 & 16 & 0 & 23 & 0 & 23 & 0 & 2 & 21 & 22 & 1 & 1 & 20 \\
17 & 16 & 0 & 23 & 0 & 23 & 0 & 0 & 23 & 23 & 0 & 21 & 0 \\
18 & 16 & 0 & 23 & 0 & 23 & 0 & 23 & 0 & 0 & 23 & 0 & 21 \\
\hline
\end{tabular}

Table 5: Confusion matrix for the $\mathcal{W}$-GLRT detector.

\begin{tabular}{lccc} 
& & \multicolumn{2}{c}{ True condition } \\
\cline { 3 - 4 } & & $\begin{array}{c}\text { Condition } \\
\text { positive }\end{array}$ & $\begin{array}{c}\text { Condition } \\
\text { negative }\end{array}$ \\
\hline Predicted & Condition positive & 1159 & 178 \\
condition & Condition negative & 371 & 614 \\
\hline & & $75.8 \%$ & $77.5 \%$ \\
& & Sensitivity & Specificity \\
\hline
\end{tabular}


to estimate the vessel roll natural frequency, it has been considered that it should be coinciding (or at least very close) to the roll spectrum peak frequency. This assumption could be made due to the ship roll dynamics, which tend to mitigate the effect of excitation which do not generate motions close to the roll natural frequency. However, when the observed roll motions are small, the roll spectrum may present additional peaks to that of the roll natural frequency, or even present a single peak shifted to the excitation frequency, thus masking the expected natural frequency value.

This issue could be addressed through the integration of systems for sea state estimation to determine the prevailing wave characteristics (e.g. (Pascoal et al., 2007; Tannuri et al., 2003; Nielsen, 2006, 2017; Iseki and Ohtsu, 2000)). Such systems would provide an estimate of the current wave frequency which could be used to improve the accuracy of the roll natural frequency selection among all the MIFs.

In the case of the $\mathcal{W}$-GLRT detector, a similar behaviour to that of the frequency estimation subsystem is observed, although the fact that its performance is not only dependent on estimated roll frequency makes it work well in most of the sea states. It adequately detects a potentially unsafe situation in real-time and the percentage of false alarms generated is generally low.

The last point of discussion is the situation awareness system. Its performance is highly related to the accuracy of the roll natural frequency estimates. So, if there is little dispersion on these data, then $\hat{\lambda}_{1}(\ln 2)^{\frac{1}{\hat{\kappa}_{1}}}$ is computed more accurately and the situation awareness system is capable of distinguishing the changes in the loading condition, showing good results. When the quality of the estimates decreases, the system becomes more insensitive, only identifying large changes in loading condition.

\section{Conclusions}

The implementation of on-board stability monitoring systems for fishing vessels could reduce the high rate of stability-related accidents. This type of systems usually requires the interaction with the crew, whose scarce insight may increase the uncertainty of the provided stability estimations.

In this work, a new method for providing stability information to the crew has been described. The approach is based on a real-time estimation of the roll natural frequency of the vessel and on the detection of possible changes in this parameter. In this way, both the stability level of the ship in 
a given sailing situation and the effects of changes in the loading condition, can be evaluated.

The first part of the method is focused on the real-time estimation of the roll natural frequency from the monitored roll motion time series. The Empirical Mode Decomposition combined with the Hilbert-Huang Transform is used to estimate the roll natural frequency. The second part consists on a detection scheme based on the Weibull distribution together with the Generalized Likelihood Ratio Test. Its purpose is to decide if the current estimates belong to a safe or to a hazardous condition, thus providing an indication of the safety of the current loading condition. Furthermore, a colour code warning produced by a situation awareness system is used to provide the stability level to the crew in a clear and understandable way.

To analyze the suitability of the entire procedure, roll time series obtained using a nonlinear mathematical model of roll motion in irregular beam waves of a medium sized stern trawler, in 6 loading conditions and 18 sea states, have been used.

The general performance of the method is promising. The frequency estimation subsystem has shown good accuracy in estimating the roll natural frequency of the ship in real-time for most of the sea states in those loading conditions with largest $G M$, and for the low stability loading conditions in those sea states with peak frequencies smaller than the expected ship roll natural frequency. However, the methodology performed less good in the conditions with smallest $G M$ and for sea states with peak frequencies larger than the natural frequency target value. A similar behaviour has been reflected in the performance of the GLRT detector, although it was able to distinguish between safe and unsafe situations even in conditions in which the performance of the EMD-HHT module was not optimal, thus increasing the number of situations in which the crew can obtain an indication of whether their current loading condition is safe or not.

\section{Acknowledgements}

This work was supported by University of A Coruña and INDITEX SA under the "Collaboration agreement between UDC and INDITEX for the internationalization of doctoral studies" [grant number 04.00.47.00.01 422D 48001]. 


\section{Appendix}

Table 6: Summary of of the roll natural frequency estimates for different loading conditions and sea states.

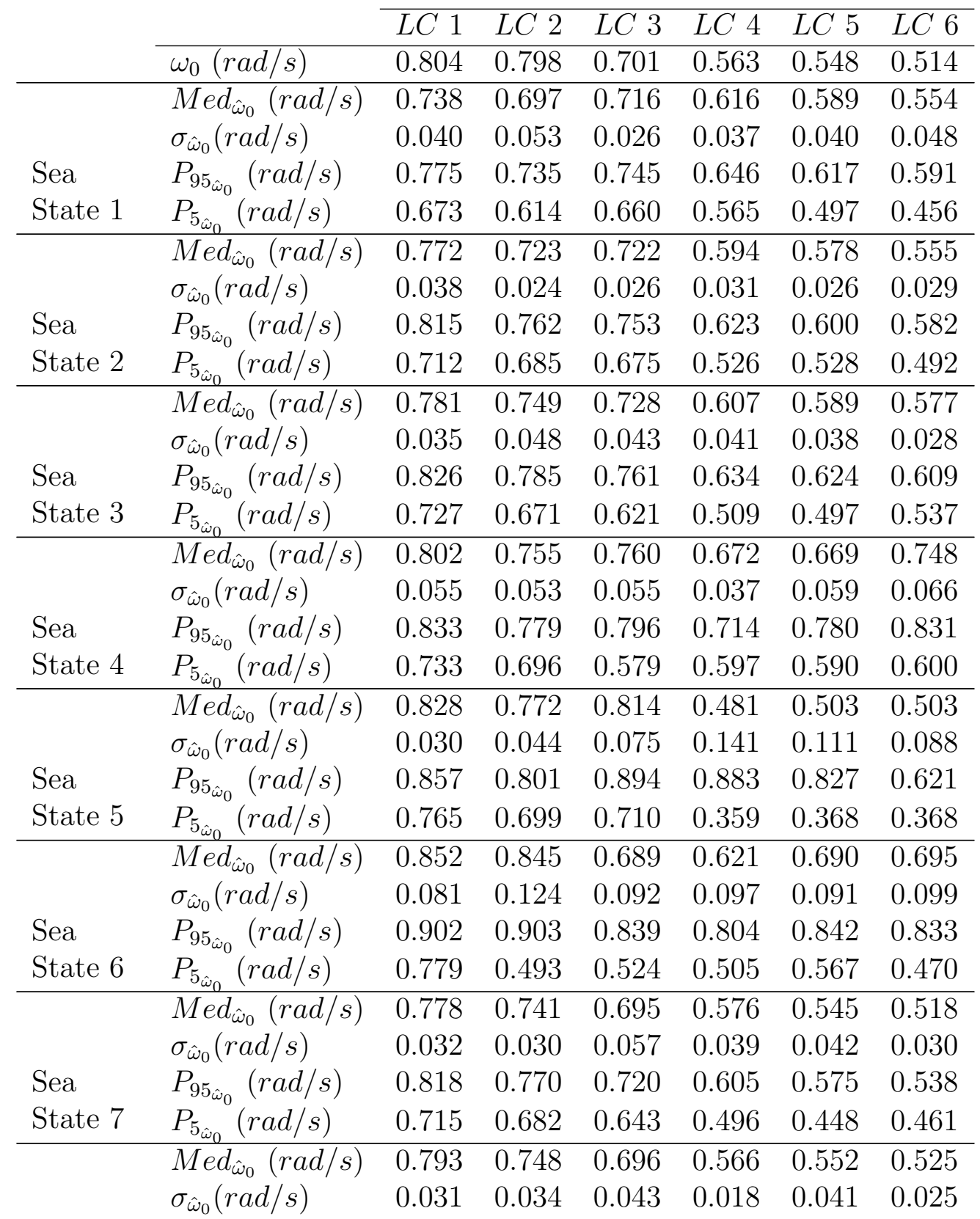

Sea

State 8 


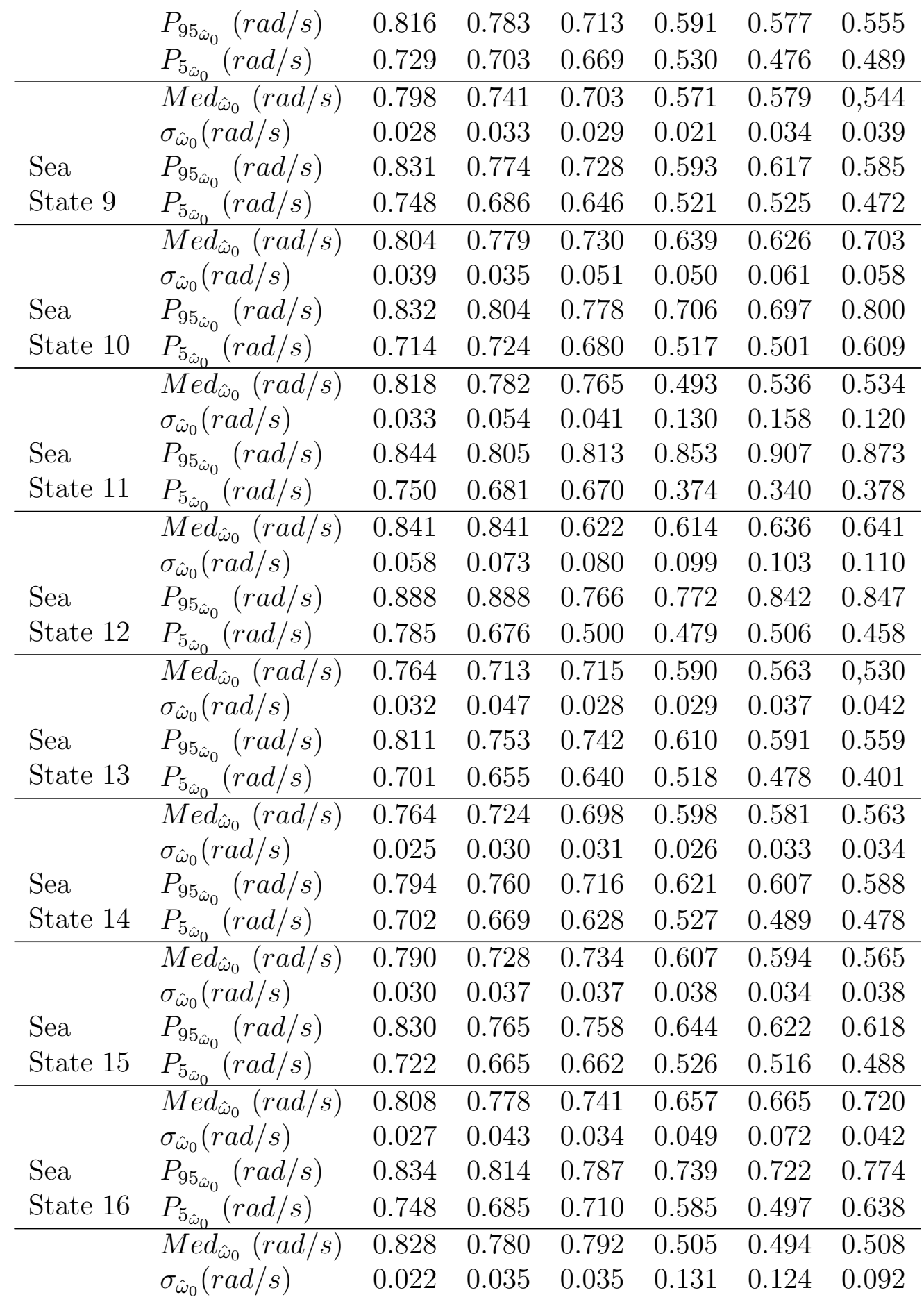

Sea

State 17 


\begin{tabular}{llllllll} 
& $P_{95_{\omega_{0}}}(\mathrm{rad} / \mathrm{s})$ & 0.856 & 0.807 & 0.825 & 0.863 & 0.843 & 0.715 \\
& $P_{5_{\hat{\omega}_{0}}}(\mathrm{rad} / \mathrm{s})$ & 0.782 & 0.715 & 0.738 & 0.363 & 0.340 & 0.417 \\
\hline & $M e d_{\hat{\omega}_{0}}(\mathrm{rad} / \mathrm{s})$ & 0.855 & 0.819 & 0.630 & 0.644 & 0.663 & 0,676 \\
& $\sigma_{\hat{\omega}_{0}}(\mathrm{rad} / \mathrm{s})$ & 0.038 & 0.045 & 0.097 & 0.117 & 0.095 & 0.107 \\
Sea & $P_{95_{\hat{\omega}_{0}}}(\mathrm{rad} / \mathrm{s})$ & 0.904 & 0.871 & 0.763 & 0.833 & 0.804 & 0.821 \\
State 18 & $P_{5_{\hat{\omega}_{0}}}(\mathrm{rad} / \mathrm{s})$ & 0.785 & 0.729 & 0.441 & 0.470 & 0.490 & 0.476 \\
\hline
\end{tabular}

\section{References}

Antaõ, P., Almeida, T., Jacinto, C., Soares, C.G., 2008. Causes of occupational accidents in the fishing sector in Portugal. Safety Science 46, 885-899. doi:10.1016/j.ssci.2007.11.007.

Bhattacharyya, R., 1978. Dynamics of marine vehicles. Wiley - Interscience, New York.

Bulian, G., Francescutto, A., 2004. A simplified modular approach for the prediction of the roll motion due to the combined action of wind, in: Proceedings of the Institution of Mechanical Engineers, Part M: Journal of Engineering for the Maritime Environment, pp. 189-212. doi:10.1243/1475090041737958.

Bulian, G., Francescutto, A., 2006. Safety and operability of fishing vessels in beam and longitudinal waves. The Transactions of The Royal Institution Of Naval Architects. Part B, International Journal of Small Craft Technology 148, 1-16.

Bulian, G., Francescutto, A., 2009. Experimental results and numerical simulations on strongly non-linear rolling of multihulls in moderate beam seas. Proceedings of the Institution of Mechanical Engineers, Part M: Journal of Engineering for the Maritime Environment 223, 189-210.

Bye, R., Lamvik, G.M., 2007. Professional culture and risk perception: Coping with danger on board small fishing boats and offshore service vessels. Reliability Engineering \& System Safety 92, 1756-1763. doi:10.1016/j.ress.2007.03.024. 
Dätig, M., Schlurmann, T., 2004. Performance and limitations of the HilbertHuang transformation (HHT) with an application to irregular water waves. Ocean Engineering 31, 1783-1834. doi:10.1016/j.oceaneng.2004.03.007.

Deakin, B., 2005. Development of simplified stability and loading information for fishermen. RINA, Royal Institution of Naval Architects International Conference - Fishing Vessels, Fishing Technology and Fisheries , 37-46.

Dickey, D.H., 2008. Analysis of Fishing Vessel Casualties A Review of Lost Fishing Vessels and Crew. Technical Report. Office of Investigations and Analysis, United States Coast Guard. Washington DC.

European Commission, 2009. Causes and circumstances of accidents at work in the EU. Technical Report. Directorate-General for Employment, Social Affairs and Equal Opportunities, European Commission. Luxembourg.

Fang, S., Blanke, M., Leira, B.J., 2015. Control Engineering Practice Mooring system diagnosis and structural reliability control for position moored vessels. Control Engineering Practice 36, 1226. URL: http://dx.doi.org/10.1016/j.conengprac.2014.11.009, doi:10.1016/j.conengprac.2014.11.009.

Fossen, T.I., Perez, T., 2009. Kalman Filtering for Positioning and Heading Control of Ships and Offshore Rigs. IEEE Control Systems Magazine CST29(6), 32-46.

Galeazzi, R., Blanke, M., Falkenberg, T., Poulsen, N.K., Violaris, N., Storhaug, G., Huss, M., 2015. Parametric roll resonance monitoring using signal-based detection. Ocean Engineering 109, 355371. URL: http://dx.doi.org/10.1016/j.oceaneng.2015.08.037, doi:10.1016/j.oceaneng.2015.08.037.

Galeazzi, R., Blanke, M., Poulsen, N.K., 2013. Early detection of parametric roll resonance on container ships. IEEE Transactions on Control Systems Technology 21, 489-503. doi:10.1109/TCST.2012.2189399.

Galeazzi, R., Perez, T., 2011. A Nonlinear Observer for Estimating Transverse Stability Parameters of Marine Surface Vessels. volume 44. IFAC. URL: http://dx.doi.org/10.3182/20110828-6-IT-1002.01474, doi:10.3182/20110828-6-IT-1002.01474. 
Ghane, M., Rasekhi Nejad, A., Blanke, M., Gao, Z., Moan, T., 2018. Condition monitoring of spar-type floating wind turbine drivetrain using statistical fault diagnosis. Wind Energy 21, 575-589. doi:10.1002/we.2179.

Grimble, M.J., Patton, R.J., Wise, D.A., 1980. Use of Kalman filtering techniques in dynamic ship-positioning systems. IEE Proceedings D - Control Theory and Applications 127, 93-102.

Gudmundsson, A., 2013. The FAO/ILO/IMO Safety Recommendations for Decked Fishing Vessels of Less than 12 metres in Length and Undecked Fishing Vessels a major milestone to improve safety for small fishing vessels, in: Proceedings of the 13th International Ship Stability Workshop, Brest, France.

Gupta, R., Kumar, A., Bahl, R., 2014. Estimation of instantaneous frequencies using iterative empirical mode decomposition. Signal, Image and Video Processing 8, 799-812. doi:10.1007/s11760-012-0305-5.

Hansen, S., Blanke, M., 2014. Diagnosis of Airspeed Measurement Faults for Unmanned Aerial Vehicles. IEEE Transactions on Aerospace and Electronic Systems 50, 224-239. doi:10.1109/TAES.2013.120420.

Holzhiiter, T., 1992. On the Robustness of Course Keeping Autopilots. IFAC Proceedings Volumes 25, 235-244. doi:10.1016/S1474-6670(17)50296-7.

Huang, N., Shen, Z., Long, S., Wu, M., SHIH, H., ZHENG, Q., Yen, N., Tung, C., Liu, H., 1998. The empirical mode decomposition and the Hilbert spectrum for nonlinear and non-stationary time series analysis, in: Proceedings of the Royal Society A: Mathematical, Physical and Engineering Sciences, pp. 903-995. doi:10.1098/rspa.1998.0193.

International Maritime Organization, 1993. Resolution A.749(18). Code on Intact Stability for all Types of Ships Covered by IMO Instruments.

International Maritime Organization, 2007. MSC.1/Circ.1228. Revised Guidance to the Master for Avoiding Dangerous Situations in Adverse Weather and Sea Conditions.

International Maritime Organization, 2008. International Code on Intact Stability (2008 IS Code). 
International Maritime Organization, 2012. Cape Town Agreement of 2012 on the Implementation of the Provisions of the Torremolinos Protocol of 1993 Relating to the Torremolinos International Convention for the Safety of Fishing Vessels, 1977.

International Towing Tank Conference, 2002. The Specialist Committee on Waves. Final Report and Recommendations to the 23rd ITTC, in: Proceedings of the 23rd International Towing Tank Conference, Venice, Italy.

Iseki, T., Ohtsu, K., 2000. Bayesian estimation of directional wave spectra based on ship motions. Control Engineering Practice 8, 215-219.

Jensen, O.C.C., Petursdottir, G., Holmen, I.M., Abrahamsen, A., Lincoln, J., 2014. A review of fatal accident incidence rate trends in fishing. International Maritime Health 65, 47-52. doi:10.5603/IMH.2014.0011.

Kay, S.M., 1998. Fundamentals of statistical signal proccessing: detection theory. Prentice-Hall, Inc., Englewood Cliffs.

Krata, P., 2008. Total Losses of Fishing Vessels Due to the Insufficient Stability. International Journal on Marine Navigation and Safety of Sea Transportation 2, 311-315.

Krüger, S., Kluwe, F., 2008. A simplified method for the estimation of the natural roll frequency of ships in heavy weather. HANSA International Maritime Journal 9.

Lazakis, I., Kurt, R.E., Turan, O., 2014. Contribution of human factors to fishing vessels accidents and near misses in the UK. Journal of Shipping and Ocean Engineering 4, 245-261. doi:10.1037/0033-3204.44.3.0.

Lucas, D.L., Case, S.L., 2018. Workrelated mortality in the US fishing industry during 20002014: New findings based on improved workforce exposure estimates. American journal of industrial medicine 61(1), 21-31.

Míguez González, M., Bulian, G., 2018. Influence of ship dynamics modelling on the prediction of fishing vessels roll response in beam and longitudinal waves. Ocean Engineering 148, 312-330. doi:10.1016/j.oceaneng.2017.11.032. 
Míguez González, M., Bulian, G., Santiago Caamaño, L., Díaz Casás, V., 2017. Towards real-time identification of initial stability from ship roll motion analysis, in: Proceedings of the 16th International Ship Stability Workshop, Belgrade, Serbia.

Míguez González, M., Caamaño Sobrino, P., Tedín Álvarez, R., Díaz Casás, V., Martínez López, A., López Peña, F., 2012. Fishing vessel stability assessment system. Ocean Engineering 41, 67-78.

Nielsen, U.D., 2006. Estimations of on-site directional wave spectra from measured ship responses. Marine Structures 19, 33-69. doi:10.1016/j.marstruc.2006.06.001.

Nielsen, U.D., 2017. A concise account of techniques available for shipboard sea state estimation. Ocean Engineering 129, 352362. URL: http://dx.doi.org/10.1016/j.oceaneng.2016.11.035, doi:10.1016/j.oceaneng.2016.11.035.

Oliveira-Goumas, B., El Houdagui, R., 2000. Information note fish 501 EN: Safety and the causes of accidents in the fisheries sector. Technical Report. European Parliament.

Pascoal, R., Guedes Soares, C., Sorensen, A.J., 2007. Ocean Wave Spectral Estimation Using Vessel Wave Frequency Motions. Journal of Offshore Mechanics and Arctic Engineering 129, 90. doi:10.1115/1.2426986.

Petursdottir, G., Hannibalsson, O., Turner, J.M.M., 2001. Safety At Sea As an Integral Part of Fisheries Management Safety At Sea As an Integral Part. FAO Fisheries Circular. No. 966 .

Pradhan, C., Gupta, A., 2017. Ship detection using Neyman-Pearson criterion in marine environment. Ocean Engineering 143, 106112. URL: http://dx.doi.org/10.1016/j.oceaneng.2017.03.008, doi:10.1016/j.oceaneng.2017.03.008.

Sagatun, S.I., Fossen, T.I., 1996. Design of a dynamic positioning system using model-based control. Control Engineering Practice 4, 359-368.

Santiago Caamaño, L., Míguez González, M., Díaz Casás, V., 2018a. Improving the safety of fishing vessels trough roll motion analysis, in: Proceedings 
of the ASME 2018 37th International Conference on Ocean Offshore and Artic Engineering, Madrid, Spain.

Santiago Caamaño, L., Míguez González, M., Díaz Casás, V., 2018b. On the feasibility of a real time stability assessment for fishing vessels. Ocean Engineering 159, 76-87. doi:10.1016/j.oceaneng.2018.04.002.

Scarponi, M., 2017. Use of the Wolfson stability guidance for appraising the operational stability of small fishing vessels, in: Proceedings of the 16th International Ship Stability Workshop, Belgrade, Serbia.

Strand, J.P., Fossen, T.I., 1999. Nonlinear passive observer design for ships with adaptive wave filtering, in: Nijmeijer, H., Fossen, T.I. (Eds.), New Directions in nonlinear observer design, Springer London, London. pp. 113-134.

Tannuri, E.A., Sparano, J.V., Simos, A.N., Da Cruz, J.J., 2003. Estimating directional wave spectrum based on stationary ship motion measurements. Applied Ocean Research 25, 243-261. doi:10.1016/j.apor.2004.01.003.

Tello, M., Ribeiro e Silva, S., Guedes Soares, C., 2011. Seakeeping performance of fishing vessels in irregular waves. Ocean Engineering 38, 763-773.

Terada, D., Hashimoto, H., Matsuda, A., Umeda, N., 2018. Direct estimation of natural roll frequency using onboard data based on a Bayesian modeling procedure, in: Proceedings of the 13th International Conference on the Stability of Ships and Ocean Vehicles, Kobe, Japan.

Terada, D., Tamashima, M., Nakao, I., Matsuda, A., 2016. Estimation of the metacentric height by using onboard monitoring roll data based on time series analysis, in: Proceedings of the 15th International Ship Stability Workshop, Stockholm, Sweden.

Wawrzynski, W., Krata, P., 2016. Method for ship's rolling period prediction with regard to non-linearity of GZ curve. Journal of theoretical and applied mechanics , 1329-1343doi:10.15632/jtam-pl.54.4.1329.

Willersrud, A., Blanke, M., Member, S., Imsland, L., Pavlov, A., 2015. Drillstring Washout Diagnosis Using Friction Estimation and Statistical Change Detection. IEEE Transactions on Control Systems Technology 23, 1886-1900. doi:10.1109/TCST.2015.2394243. 
Wolfson Unit, 2004. Research project 530. Simplified presentation of FV statibility information - phase 1. Final report. Technical Report 4.

Womack, J., 2003. Small commercial fishing vessel stability analysis: Where are we now? Where are we going? Marine Technology 40, 296-302.

Xie, H., Wang, Z., 2006. Mean frequency derived via HilbertHuang transform with application to fatigue EMG signal analysis. Computer Methods and Programs in Biomedicine 82, 114-120. doi:10.1016/j.cmpb.2006.02.009. 